\title{
EFEITO DE DIFERENTES NÍVEIS DE EXPLORAÇÃO E DE TRATAMENTOS SILVICULTURAIS SOBRE A DINÂMICA DA FLORESTA REMANESCENTE
}

\author{
Celso Paulo de Azevedo*, Carlos Roberto Sanquetta**, José Natalino Macedo Silva***, \\ Sebastião do Amaral Machado**** \\ *Eng. Florestal, Dr., Embrapa Amazônia Ocidental - celso@cpaa.embrapa.br
**Eng. Florestal,Dr., Depto. de Ciências Florestais, UFPR - sanquetta@ufpr.br
***Eng. Florestal, Ph.D., Embrapa Amazônia Oriental - natalino.silva@cgiar.org \\ ****Eng. Florestal, Ph.D., Depto. de Ciências Florestais, UFPR, Pesquisador 1A do CNPq - samachado@ufpr.br
}

Recebido para publicação: 21/07/2007 - Aceito para publicação: 23/10/2007

\begin{abstract}
Resumo
Este estudo foi conduzido com dados do Projeto Bom Manejo (Embrapa/CIFOR/ITTO) - Manejo sustentável de florestas de produção em escala comercial na Amazônia brasileira, desenvolvido pela Embrapa Amazônia Oriental, em área da Companhia Florestal Monte Dourado (Jari), município de Vitória do Jari, Estado do Amapá. O delineamento é em blocos ao acaso, com três repetições. Apresenta-se uma avaliação da dinâmica da vegetação com dap $\geq 20 \mathrm{~cm}$, submetida a três intensidades de exploração (corte de $15 \%, 25 \%$ e $35 \%$ do volume total das árvores com mais de 60 $\mathrm{cm}$ de dap), combinadas com quatro níveis de redução de área basal $(0 \%, 30 \%, 50 \%$ e $70 \%$ da área basal original) por anelamento. $\mathrm{Na}$ área experimental, estão locadas 40 parcelas permanentes de 1 ha (36 exploradas e 4 não-exploradas). O povoamento foi medido em 1984, explorado em 1985 e remedido em 1986, 1988, 1990, 1994, 1996 e 2004. Concluiu-se que a exploração de impacto reduzido foi boa para a floresta, mas não, necessariamente, estimulou o crescimento, e deve levar a ciclos de corte maiores do que os recomendados trinta anos.

Palavras-chave: Medições repetidas no tempo; incremento; ingresso; mortalidade; floresta tropical.
\end{abstract}

\begin{abstract}
Effect of different logging intensities and treatments on the dynamics of the remaining forest. This study was conducted with data from Projeto Bom Manejo (Embrapa/CIFOR/ITTO) - Sustainable management of production forests in commercial scale in the Brazilian Amazon, carried out by Embrapa Eastern Amazon in Monte Dourado Forest Company (Jari) area, Vitória do Jari, State of Amapá, Brazil. The experimental design was randomized blocks with three replications. An evaluation of the dynamics of the vegetation with $\mathrm{DBH} \geq 20 \mathrm{~cm}$ is presented, under three logging intensities (extraction of $15 \%, 25 \%$ and $35 \%$ ) of the total volume of trees with more than $60 \mathrm{~cm}$ of $\mathrm{dbh}$ were tested, followed of four levels of basal area reduction $(0 \%, 30 \%, 50 \%$ and $70 \%$ of the original basal area). The forest development was monitored through 40 permanent sample plots of 1 ha each (36 logged and 4 unlogged). The plots were measured in 1984, logged in 1985 and remeasured in 1986, 1988, 1990, 1994, 1996 and 2004. The results showed that reduced impact logging was good for the forest, but not, necessarily, it stimulated the growth and it must take longer cutting cycles than the recommended thirty years.

Keywords: Time repeated-measures analysis; ingrowth; mortality; increment; tropical forest.
\end{abstract}

\section{INTRODUÇ̃̃O}

Experiências silviculturais conduzidas na Amazônia inspiraram dois sistemas de manejo para a região, o Sistema Silvicultural Brasileiro para Florestas de Terra Firme da Amazônia (SSB), sugerido por Yared et al. (2000), e o sistema Seleção de Espécies Listadas (SEL), desenvolvido pelo Instituto Nacional de Pesquisas da Amazônia (INPA) (HIGUCHI et al., 1991). Esses dois sistemas são do tipo policíclico e 
de uso múltiplo, sendo análogos aos princípios dos sistemas precursores - Malaio e de Shelterwood Tropical -, que utilizam a regeneração natural para garantir ciclos de corte subseqüentes.

Para avaliar o caráter dinâmico do crescimento de uma floresta, por exemplo, após uma intervenção exploratória dos recursos madeireiros, faz-se necessário proceder ao monitoramento, o qual é definido como o instrumento de avaliação do caráter dinâmico da comunidade e de uma série de variáveis indispensáveis para a definição do manejo (QUEIROZ, 1998; PÉLLICO NETTO; BRENA, 1997).

Esse procedimento é conhecido como Inventário Florestal Contínuo (IFC) e corresponde a efetuar medições na floresta repetidas vezes no tempo, ou seja, medições em várias ocasiões. Tal procedimento resulta na obtenção de uma série de informações fundamentais aos manejadores, como crescimento, ingresso, mortalidade, ciclo de corte, sucessão e densidade de estoque, entre outros.

A dinâmica da floresta pode resumir-se ao entendimento do comportamento das taxas de crescimento, de recrutamento e de mortalidade, em condições naturais e sob manejo. Essas informações são fundamentais para a definição do ciclo de corte do manejo florestal, da intensidade de colheita e para a prescrição de tratamentos silviculturais nas florestas manejadas (ROCHA, 2001).

As taxas de crescimento podem ser aceleradas pelos tratamentos silviculturais, que envolvem dois tipos: i) liberação ou desbaste seletivo, que consiste na remoção de indivíduos competidores, nãodesejáveis, cujas copas estejam competindo por luz com as copas das árvores de espécies selecionadas para a próxima colheita; ou ii) refinamento ou desbaste sistemático, que consiste na redução da área basal de espécies não-desejáveis, visando diminuir a competição no povoamento de forma geral.

Segundo Jardim (1996), em termos silviculturais, a abertura do dossel pode ser feita de duas maneiras principais: através do abate de árvores, na exploração florestal ou nos desbastes, e através de técnicas de anelamento de árvores, com ou sem envenenamento. No entanto, a técnica de anelamento é pouco eficiente na eliminação de indivíduos de algumas espécies, principalmente aquelas com reentrâncias no fuste (COSTA et al., 2001).

O presente trabalho teve como objetivo analisar o efeito de diferentes níveis de exploração e de tratamentos silviculturais sobre a dinâmica (mortalidade, ingresso, crescimento em número de árvores, em diâmetro, em área basal e volume e incrementos em diâmetro, em área basal e volume) do povoamento florestal remanescente.

\section{MATERIAL E MÉTODO}

\section{Localização e caracterização da área}

Este estudo foi conduzido com dados do Projeto Bom Manejo (Embrapa Amazônia Oriental /CIFOR/ITTO). O experimento foi iniciado em 1983, em uma área de 500 ha, na Companhia Florestal Monte Dourado (Jari), na localidade Morro do Felipe, Vitória do Jari (AP), coordenadas $52^{\circ} 20^{\prime} \mathrm{O}$ e $00^{\circ} 55^{\prime} \mathrm{S}$, a uma altitude de aproximadamente $150 \mathrm{~m}$. O clima nessa região é do tipo Ami, pela classificação de Köppen. A precipitação média anual alcança $2.234 \mathrm{~mm}$. A temperatura média anual é de $25,8{ }^{\circ} \mathrm{C}$ e a vegetação é do tipo Floresta Ombrófila Densa. Os solos são do tipo Latossolo Amarelo Distrófico, com textura argilosa pesada.

\section{Delineamento experimental}

Em 400 há, foram plotados três blocos de 48 ha. Todos os blocos têm bordadura e foram deixadas faixas entre um bloco e outro e entre os blocos e as estradas permanentes. Dentro dos blocos experimentais, a exploração foi feita em faixas, sendo cada faixa (200 m x $800 \mathrm{~m})$ com uma intensidade diferente. As alternativas de manejo testadas foram combinações de intensidade de exploração com intensidade de desbastes. As intensidades de exploração $(15 \%$ - intensidade leve, $25 \%$ - intensidade média e $35 \%$ - intensidade pesada) de baixo impacto foram representadas por porcentagens do volume extraído em relação ao volume total da floresta, a partir do diâmetro à altura do peito (dap) de $60 \mathrm{~cm}$. Elas foram combinadas com quatro tipos de intensidades de desbastes, o desbaste sistemático nas intensidades de $0 \%, 30 \%, 50 \%$ e $70 \%$ de redução da área basal original por anelamento de árvores do dossel (Tabela 1 e Figura 1). 100 ha foram mantidos como testemunha.

\section{Monitoramento da floresta}

$\mathrm{Na}$ área experimental, foram locadas 40 parcelas permanentes de 1 hectare - trinta e seis em área explorada e quatro em área não-explorada. A primeira medição foi realizada em 1984, a exploração em 
1985 e as remedições em 1986, 1988, 1990, 1994, 1996 e 2004 (Figura 1). Todas as árvores com diâmetro maior ou igual a $20,0 \mathrm{~cm}$ foram medidas. A metodologia empregada foi desenvolvida e disponibilizada por Silva; Lopes (1984).

Tabela 1. Descrição dos tratamentos.

Table 1. Description of the treatments.

\begin{tabular}{|c|c|}
\hline Tratamento & Descrição \\
\hline $\mathrm{T}_{1}$ & $\begin{array}{l}\text { Exploração de } 15 \% \text { do volume total das árvores de dap }>60 \mathrm{~cm} \text {, sem redução da área basal após a } \\
\text { exploração. }\end{array}$ \\
\hline $\mathrm{T}_{2}$ & $\begin{array}{l}\text { Exploração de } 15 \% \text { do volume total das árvores de dap }>60 \mathrm{~cm} \text {, com redução de } 30 \% \text { da área basal } \\
\text { original através de tratos silviculturais. }\end{array}$ \\
\hline $\mathrm{T}_{3}$ & $\begin{array}{l}\text { Exploração de } 15 \% \text { do volume total das árvores de dap }>60 \mathrm{~cm} \text {, com redução de } 50 \% \text { da área basal } \\
\text { original através de tratos silviculturais. }\end{array}$ \\
\hline $\mathrm{T}_{4}$ & $\begin{array}{l}\text { Exploração de } 15 \% \text { do volume total das árvores de dap }>60 \mathrm{~cm} \text {, com redução de } 70 \% \text { da área basal } \\
\text { original através de tratos silviculturais. }\end{array}$ \\
\hline $\mathrm{T}_{5}$ & $\begin{array}{l}\text { Exploração de } 25 \% \text { do volume total das árvores de dap }>60 \mathrm{~cm} \text {, sem redução da área basal após a } \\
\text { exploração. }\end{array}$ \\
\hline $\mathrm{T}_{6}$ & $\begin{array}{l}\text { Exploração de } 25 \% \text { do volume total das árvores de dap }>60 \mathrm{~cm} \text {, com redução de } 30 \% \text { da área basal } \\
\text { original através de tratos silviculturais. }\end{array}$ \\
\hline $\mathrm{T}_{7}$ & $\begin{array}{l}\text { Exploração de } 25 \% \text { do volume total das árvores de dap }>60 \mathrm{~cm} \text {, com redução de } 50 \% \text { da área basal } \\
\text { original através de tratos silviculturais. }\end{array}$ \\
\hline $\mathrm{T}_{8}$ & $\begin{array}{l}\text { Exploração de } 25 \% \text { do volume total das árvores de dap }>60 \mathrm{~cm} \text {, com redução de } 70 \% \text { da área basal } \\
\text { original através de tratos silviculturais. }\end{array}$ \\
\hline $\mathrm{T}_{9}$ & $\begin{array}{l}\text { Exploração de } 35 \% \text { do volume total das árvores de dap }>60 \mathrm{~cm} \text {, sem redução da área basal após a } \\
\text { exploração. }\end{array}$ \\
\hline $\mathrm{T}_{10}$ & $\begin{array}{l}\text { Exploração de } 35 \% \text { do volume total das árvores de dap }>60 \mathrm{~cm} \text {, com redução de } 30 \% \text { da área basal } \\
\text { original através de tratos silviculturais. }\end{array}$ \\
\hline $\mathrm{T}_{11}$ & $\begin{array}{l}\text { Exploração de } 35 \% \text { do volume total das árvores de dap }>60 \mathrm{~cm} \text {, com redução de } 50 \% \text { da área basal } \\
\text { original através de tratos silviculturais. }\end{array}$ \\
\hline $\mathrm{T}_{12}$ & $\begin{array}{l}\text { Exploração de } 35 \% \text { do volume total das árvores de dap }>60 \mathrm{~cm} \text {, com redução de } 70 \% \text { da área basal } \\
\text { original através de tratos silviculturais. }\end{array}$ \\
\hline
\end{tabular}

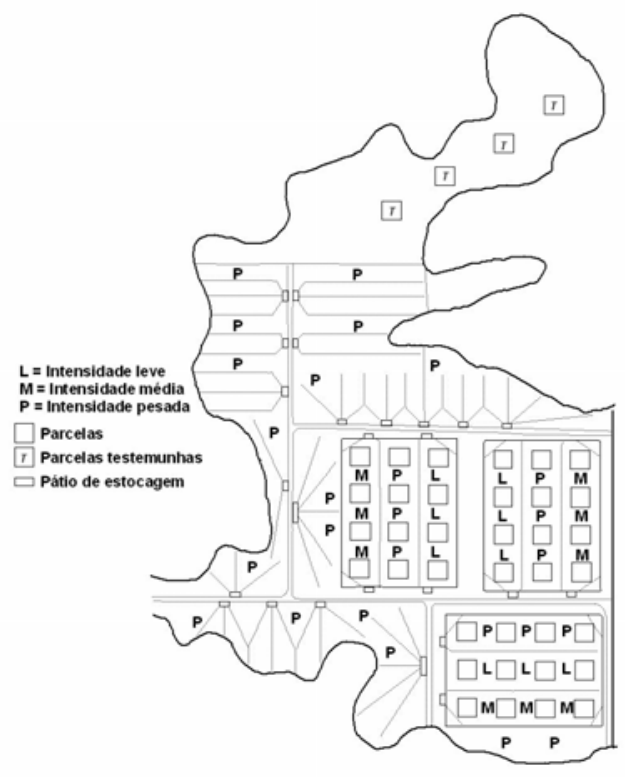

Figura 1. Croqui do experimento ( $\mathrm{L}$ - intensidade leve; $\mathrm{M}$ - intensidade média; $\mathrm{P}$ - intensidade pesada). Figure 1. Layout of the experiment (L - light intensity; M - mean intensity; P - heavy intensity). 


\section{Dinâmica da floresta}

A dinâmica sobre os tratamentos foi caracterizada com base na mudança de densidade, de área basal e de volume. O volume das árvores foi estimado pela equação abaixo (SILVA; ARAÚJO, 1984; SILVA et al., 1984):

$$
V=\left\{\begin{array}{l}
-0,0994+0,00091941 D A P^{2} \quad R^{2}=0,96 \quad S_{y x}(\%)=12 \text { se } D A P<45 \mathrm{~cm} \\
e^{(-7,6281+2,1809 \cdot \ln (D A P))} \quad R^{2}=0,84 \quad S_{y x}(\%)=16 \quad \text { se } D A P \geq 45 \mathrm{~cm}
\end{array}\right.
$$

A fórmula para o cálculo do incremento periódico anual foi a seguinte:

Crescimento $_{21}=D A P_{2}-D A P_{1}$

Nanos $_{21}=A N O_{2}-A N O_{1}$

Nmes $_{21}=M E S_{2}-M E S_{1}$

Intervalo ano $_{21}=$ Nanos $_{21}+\frac{\text { Nmes }_{21}}{12}$

$I P A_{21}=\frac{\text { Crescimento }_{21}}{\text { Intervalo } \text { ano }_{21}}$

$D A P_{I}$ : diâmetro no início do período

$D A P_{2}$ : diâmetro no final do período

Crescimento $_{21}$ : crescimento em diâmetro do período

$A N O_{I}$ : ano no início do período

$A N O_{2}$ : ano no final do período

$\operatorname{Nanos}_{21}$ : número de anos do período

$M e s_{1}$ : mês no início do período

$M e s_{2}$ : mês no final do período

Mês $s_{21}$ : número de meses do período

Intervalo ano $_{21}$ : intervalo em anos do período

$I P A_{21}$ : incremento periódico anual

Expressões semelhantes a essas foram utilizadas para os cálculos dos incrementos periódicos anuais em diâmetro, área basal $(\mathrm{G} / \mathrm{ha})$ e volume $(\mathrm{V} / \mathrm{ha})$, para todos os intervalos de medições. Pellico Netto; Brena (1997) usam o termo mudança para designar as diferenças entre dois inventários. Entretanto, o termo incremento foi mantido, por tratar-se da designação mais usual e conhecida.

A taxa anual de mortalidade foi calculada pelo modelo logarítmico utilizado por Lieberman; Lieberman (1987) e Swaine et al. (1987):

$m=100\left(\frac{\ln N_{0}-\ln N_{1}}{t}\right)$

$m=$ taxa anual de mortalidade

$N_{0}=$ número de indivíduos na amostra inicial

$N_{l}=$ número de indivíduos na amostra final

$\ln =$ logaritmo neperiano

$t=$ tempo de observações em anos

A meia vida da floresta, ou o tempo estimado para que a população seja reduzida à metade (SWAINE et al., 1987), foi obtido por:

$$
t_{0,5}=\frac{\ln (0,5)}{\ln (1-m)}
$$

$t_{0,5}=$ meia vida

$\ln$ e $m=$ como definido

O ingresso relativo foi obtido dividindo-se o número de árvores que ingressaram no período pelo número de árvores no início do referido período, multiplicando esse resultado por 100.

\section{Análise de variância de dados repetidos no tempo - medições repetidas}

As análises visaram avaliar se os crescimentos em diâmetro, em área basal e em volume e a mortalidade, o recrutamento e os incrementos periódicos anuais em diâmetro, em área basal e em volume 
foram influenciados pelas diferentes intensidades de exploração, com o passar do tempo (VON ENDE, 2001; MOSER et at., 1990). A análise do experimento obedeceu ao seguinte modelo experimental:

$$
X_{i j k l}=\mu+R_{i}+T_{j}+R T_{i j}+B_{k}+R B_{i k}+T B_{j k}+R T B_{i j k}+\varepsilon_{m(i j k)}
$$

$R=$ bloco $\quad \mathrm{i}=1, \ldots, \mathrm{r}=3$.

$T=$ tratamento (IE) $\quad \mathrm{j}=1, \ldots, \mathrm{p}=12$.

$B=$ tempo $(\mathrm{T}) \quad \mathrm{k}=1, \ldots,=7$

$X_{i j k}=$ a observação correspondente ao i-ésimo bloco, j-ésimo tratamento e k-ésimo tempo

$\mu=$ média geral comum a todas as observações

$R_{i}=$ efeito do i-ésimo bloco - fator aleatório

$T_{j}=$ efeito do j-ésimo tratamento - fator fixo

$B_{k}=$ efeito do k-ésimo tempo - fator fixo

$\varepsilon_{m(i j k)}=$ erro aleatório associado à observação $\mathrm{X}_{\mathrm{ijk}}-$ fator aleatório

Os outros termos são padrões para as interações entre os principais fatores $R, T$ e $B$.

A análise de variância de medidas repetidas foi conduzida usando-se o programa SAS 8.02 licenciado para a Embrapa. As comparações das médias pelo teste Scott-Knott (SCOTT; KNOTT, 1974) foram executadas com o auxílio do programa GENES/UFV (CRUZ, 2001).

\section{RESULTADOS E DISCUSSÃO}

\section{Povoamento total e comercial líquido}

Para o povoamento total líquido, levando em consideração o F corrigido (G-G), a significância estatística do efeito do tempo indica que a área basal, o volume, o número de árvores e o diâmetro médio $(\mathrm{p}<0,0001)$ variam significativamente com o passar do tempo (Tabela 2). Com base também nas probabilidades apresentadas nessa tabela, as interações tempo*tratamento para a área basal $(p=0,0094)$, volume $(\mathrm{p}=0,0102)$, número de árvores $(\mathrm{p}=0,3800)$ e diâmetro médio $(0,5079)$ são estatisticamente significativas nos dois primeiros casos e não-significativas nos últimos, mostrando que a aplicação dos tratamentos influenciou a área basal e o volume com o passar do tempo. Não foram encontradas diferenças estatísticas significativas entre os tratamentos para as variáveis número de árvores total $\mathrm{e}$ número de árvores comercial, considerando todo os períodos.

Oliveira (2005) encontrou diferenças significativas para essas duas variáveis, vinte e um anos após a exploração, quando as médias dos tratamentos cujas intensidades de exploração foram mais pesadas foram iguais entre si e, estatisticamente, superiores aos demais tratamentos.

Para a área basal de todas as espécies, só foram detectadas diferenças significativas logo após a exploração e nos dois últimos períodos, em conseqüência da aplicação dos tratamentos silviculturais (Tabela 2). O teste de Scott-Knott, no primeiro momento, discriminou os tratamentos leves dos demais, e no segundo momento, discriminou os tratamentos que não sofreram intervenção pós-colheita dos outros (Tabela 3). Não foram encontradas diferenças estatísticas significativas entre os tratamentos para a variável área basal de espécies comerciais, durante todo o período de acompanhamento. O comportamento do volume, ao longo do período de acompanhamento, seguiu a mesma tendência da área basal.

Neste experimento, a capacidade de resiliência da floresta mostrou-se mais eficiente para a recuperação da abundância, quando comparada à área basal e o volume. Entretanto, dezenove anos após a colheita de madeira, somente metade dos tratamentos apresentaram número de árvores superior ao encontrado antes da intervenção. Para as espécies de valor comercial, todos os tratamentos apresentaram valores inferiores aos originais, resultados diferentes dos obtidos por Oliveira (2005).

Aplicando três níveis de exploração combinados com tratamentos de refinamento, tratamento leve (exploração de $15 \mathrm{~m}^{3} \cdot \mathrm{ha}^{-1}$ ), tratamento médio (exploração de $23 \mathrm{~m}^{3} \cdot \mathrm{ha}^{-1}$ mais a redução da área basal para $18 \mathrm{~m}^{2} \cdot \mathrm{ha}^{-1}$ ) e tratamento pesado (exploração de $46 \mathrm{~m}^{3} \cdot \mathrm{ha}^{-1}$ mais a redução da área basal para 14 $\mathrm{m}^{2} \cdot \mathrm{ha}^{-1}$ ), após 20 anos de acompanhamento, Dekker; De Graaf (2003) concluíram que o tratamento médio foi o que apresentou a maior densidade de regeneração, contudo, os autores discutem a significância estatística entre os diferentes níveis de intervenções para cada repetição.

A área basal, o volume, o número de árvores e o diâmetro médio das árvores comerciais variaram significativamente somente em função do tempo. Na figura 2, nota-se claramente, pelo paralelismo das curvas, que não houve efeito significativo dos tratamentos sob essas variáveis. Observase, também, que os valores obtidos se encontram abaixo dos valores originais. 
Tabela 2. ANAVA para medidas repetidas no tempo para área basal, volume, número de árvores e diâmetro médio para o povoamento total líquido (parcela subdividida).

Table 2. Repeated-measures ANAVA for basal area, volume, number of trees and mean diameter for the net total stand for a split-plot design.

\begin{tabular}{|c|c|c|c|c|c|c|c|}
\hline \multicolumn{8}{|c|}{ Área Basal (m²/ha) } \\
\hline \multirow{2}{*}{$\begin{array}{l}\text { Parcela } \\
\text { F. variação }\end{array}$} & \multirow[b]{2}{*}{ GL } & \multirow[b]{2}{*}{ SQ } & \multirow[b]{2}{*}{$\mathbf{Q M}$} & \multirow[b]{2}{*}{$\mathbf{F}$} & \multirow[b]{2}{*}{$\operatorname{Pr}>\mathbf{F}$} & \multicolumn{2}{|c|}{ Pr $>$ F Corrigida } \\
\hline & & & & & & $\mathbf{G}-\mathbf{G}$ & $\mathbf{H}-\mathbf{F}$ \\
\hline Bloco & 2 & 114,9225511 & 57,4612755 & 2,71 & 0,0885 & & \\
\hline Tratamento & 11 & 598,3177527 & 54,3925230 & 2,57 & 0,0287 & & \\
\hline Erro & 22 & 465,8843242 & 21,1765602 & & & & \\
\hline \multicolumn{8}{|l|}{ Subparcela } \\
\hline Tempo & 6 & 297,3404211 & 49,5567369 & 52,23 & $<0,0001$ & $<0,0001$ & $<0,0001$ \\
\hline Tempo $*$ Bloco & 12 & 12,6093606 & 1,0507801 & 1,11 & 0,3594 & 0,3674 & 0,3635 \\
\hline Tempo $*$ Tratamento & 66 & 128,8557253 & 1,9523595 & 2,06 & 0,0002 & 0,0094 & 0,0008 \\
\hline \multirow[t]{3}{*}{ Erro (tempo) } & 132 & 125,2422070 & 0,9488046 & & & & \\
\hline & \multicolumn{3}{|c|}{ Greenhouse-Geisser Epsilon } & \multicolumn{2}{|c|}{0,4444} & & \\
\hline & \multicolumn{3}{|c|}{ Huynh - Feldt Epsilon } & \multicolumn{2}{|c|}{0,8103} & & \\
\hline
\end{tabular}

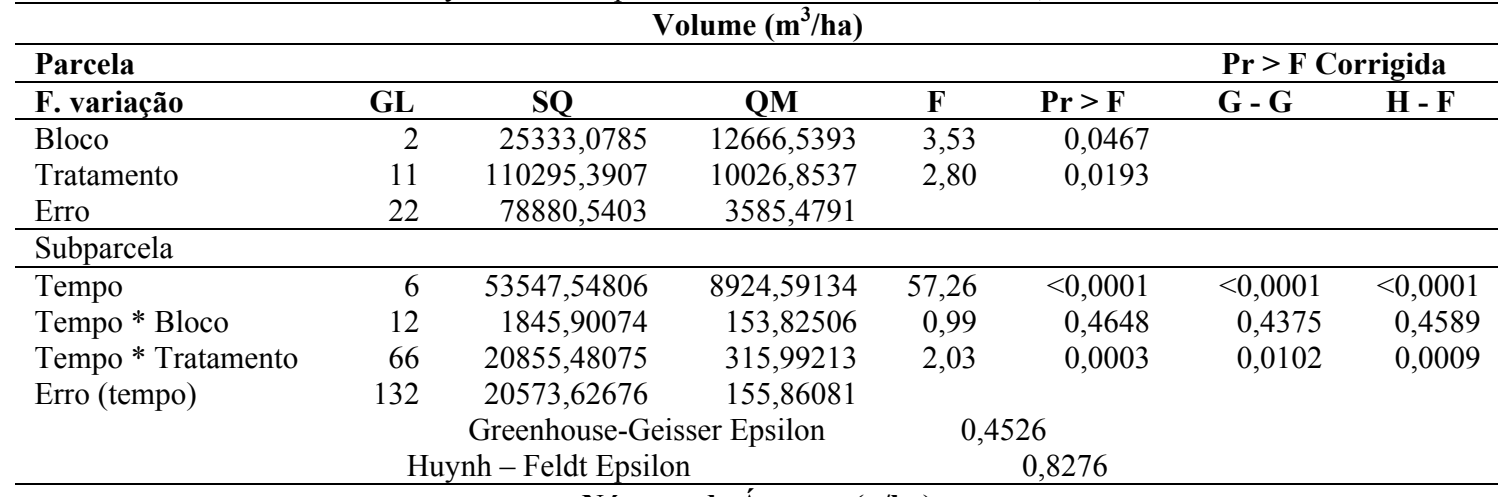

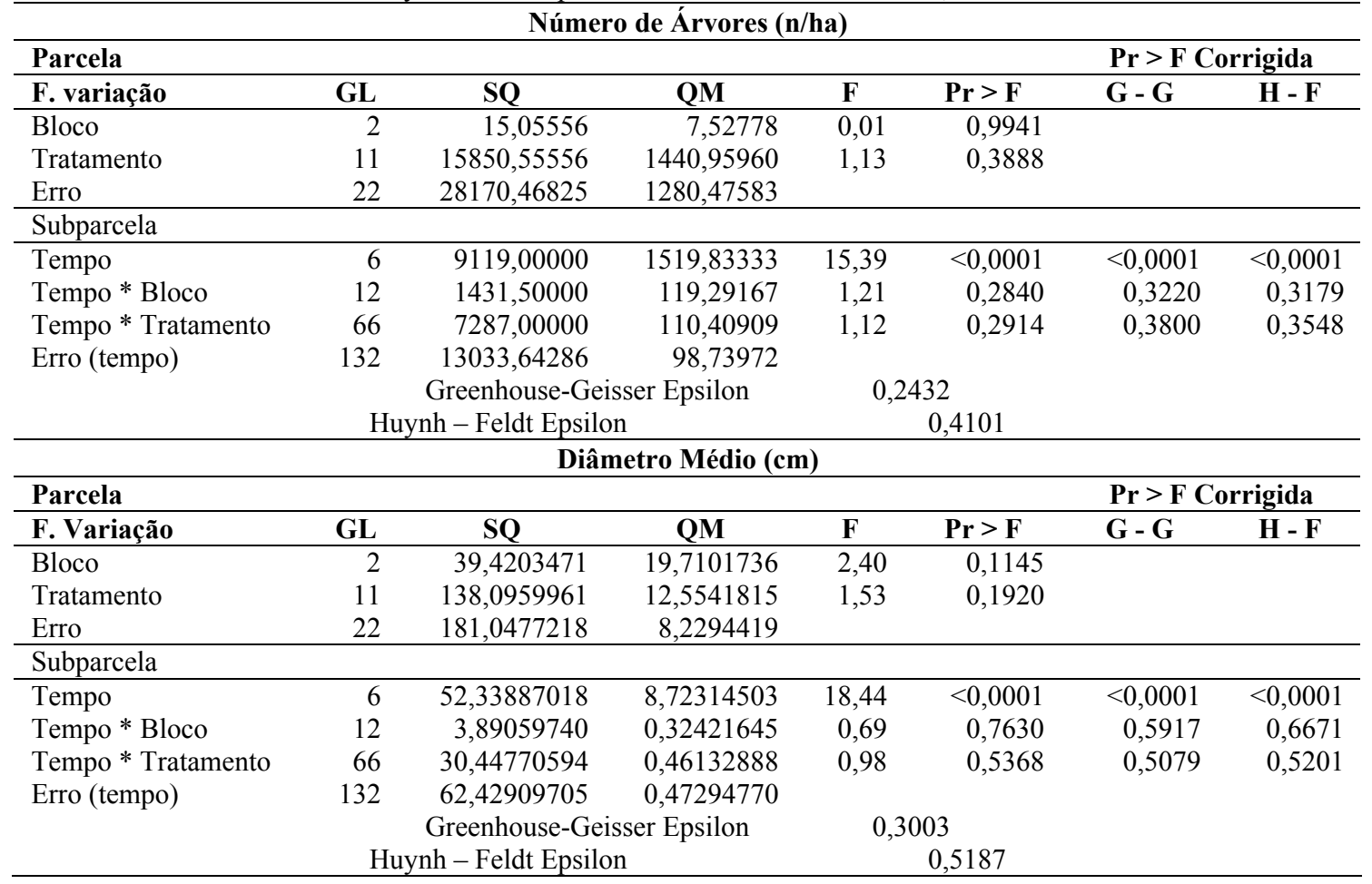

Para o povoamento total e comercial, as taxas de ingresso, os tempos de meia-vida e os balanços 
líquidos variaram significativamente somente em função do tempo $(\mathrm{p}<0,0001)$. As taxas de mortalidade comercial e total variaram significativamente em função do tempo $(\mathrm{p}<0,0001)$ e da interação tempo*tratamento $-\mathrm{p}=0,0237$ ) e $\mathrm{p}=0,0022$, respectivamente.

Tabela 3. Área basal e volume por tratamento para os anos de monitoramento, para o povoamento total líquido. (Médias seguidas da mesma letra, para cada ano, não diferem entre si ao nível de 5\% pelo teste de Scott-Knott).

Table 3. Basal area and volume per treatment for the years of monitoring, for the net total stand (Mean followed from the same letter, for each year, do not differ between itself at the level of 5\% by the test of Scott-Knott).

\begin{tabular}{|c|c|c|c|c|c|c|c|c|c|c|c|c|c|c|}
\hline \multirow{2}{*}{ Trat. } & \multicolumn{14}{|c|}{ Área basal (m²/ha) } \\
\hline & 1984 & & 1986 & & 1988 & & 1990 & & 1994 & & 1996 & & 2004 & \\
\hline 1 & 24,1122 & $\bar{a}$ & 22,0758 & $\bar{a}$ & 21,8486 & $\mathrm{a}$ & 20,9298 & $\bar{a}$ & 21,9906 & $\bar{a}$ & 22,3384 & $\mathrm{a}$ & 23,8647 & $\mathrm{a}$ \\
\hline 9 & 23,1440 & $\mathrm{a}$ & 19,2389 & $\mathrm{~b}$ & 19,1761 & $\mathrm{a}$ & 19,2323 & $\mathrm{a}$ & 20,5742 & $\mathrm{a}$ & 20,9182 & $\mathrm{a}$ & 22,5985 & $\mathrm{a}$ \\
\hline 5 & 23,1621 & $\mathrm{a}$ & 19,5309 & $\mathrm{~b}$ & 19,4657 & $\mathrm{a}$ & 19,1432 & $\mathrm{a}$ & 20,3524 & $\mathrm{a}$ & 20,7580 & $\mathrm{a}$ & 22,3425 & $\mathrm{a}$ \\
\hline 3 & 24,9614 & $\mathrm{a}$ & 23,8067 & $\mathrm{a}$ & 23,8495 & $\mathrm{a}$ & 23,8830 & $\mathrm{a}$ & 24,7347 & $\mathrm{a}$ & 23,1023 & $\mathrm{a}$ & 22,1106 & $\mathrm{a}$ \\
\hline 12 & 22,1289 & $\mathrm{a}$ & 17,7838 & $\mathrm{~b}$ & 18,1027 & $\mathrm{a}$ & 18,1867 & $\mathrm{a}$ & 19,3481 & $\mathrm{a}$ & 19,0786 & $\mathrm{~b}$ & 20,6675 & $\mathrm{~b}$ \\
\hline 2 & 22,6958 & $\mathrm{a}$ & 21,4453 & $\mathrm{a}$ & 21,2108 & $\mathrm{a}$ & 20,9897 & $\mathrm{a}$ & 21,6585 & $\mathrm{a}$ & 19,7729 & $\mathrm{~b}$ & 20,3259 & $\mathrm{~b}$ \\
\hline 4 & 21,5777 & $\mathrm{a}$ & 19,2362 & $\mathrm{~b}$ & 19,3072 & $\mathrm{a}$ & 19,6929 & $\mathrm{a}$ & 20,0276 & $\mathrm{a}$ & 19,0957 & $\mathrm{~b}$ & 20,3120 & $\mathrm{~b}$ \\
\hline 7 & 22,9792 & $\mathrm{a}$ & 20,4964 & $\mathrm{a}$ & 20,3314 & $\mathrm{a}$ & 20,1561 & $\mathrm{a}$ & 20,6564 & $\mathrm{a}$ & 20,1706 & $\mathrm{a}$ & 19,3750 & $\mathrm{~b}$ \\
\hline 8 & 23,4330 & $\mathrm{a}$ & 19,1010 & b & 19,2566 & $\mathrm{a}$ & 19,5152 & $\mathrm{a}$ & 20,6344 & $\mathrm{a}$ & 18,8919 & $\mathrm{~b}$ & 19,3260 & $\mathrm{~b}$ \\
\hline 11 & 22,0129 & $\mathrm{a}$ & 18,4602 & $\mathrm{~b}$ & 18,5945 & $\mathrm{a}$ & 17,2194 & $\mathrm{a}$ & 17,6875 & $\mathrm{a}$ & 17,2760 & $\mathrm{~b}$ & 19,0084 & $\mathrm{~b}$ \\
\hline 6 & 21,1917 & $\mathrm{a}$ & 17,5861 & $\mathrm{~b}$ & 17,8531 & $\mathrm{a}$ & 17,7094 & $\mathrm{a}$ & 18,3931 & $\mathrm{a}$ & 17,5363 & $\mathrm{~b}$ & 18,9523 & $\mathrm{~b}$ \\
\hline 10 & 22,9345 & $\mathrm{a}$ & 17,6078 & $\mathrm{~b}$ & 17,7000 & $\mathrm{a}$ & 17,6561 & $\mathrm{a}$ & 18,6277 & $\mathrm{a}$ & 17,4603 & $\mathrm{~b}$ & 17,5248 & $\mathrm{~b}$ \\
\hline Méc & 22,8611 & & 19,6974 & & 19,7247 & & 19,5261 & & 20,39 & & 19,699 & & 20,5340 & \\
\hline
\end{tabular}

\begin{tabular}{|c|c|c|c|c|c|c|c|c|c|c|c|c|c|c|}
\hline \multirow{2}{*}{ Trat. } & \multicolumn{14}{|c|}{ Volume $\left(m^{3} / h a\right)$} \\
\hline & 1984 & & 1986 & & 1988 & & 1990 & & 1994 & & 1996 & & 2004 & \\
\hline 1 & 292,17 & $\mathrm{a}$ & 265,29 & $\mathrm{a}$ & 262,91 & $\mathrm{a}$ & 251,64 & $\mathrm{a}$ & 264,37 & $\mathrm{a}$ & 269,03 & $\mathrm{a}$ & 287,35 & $a$ \\
\hline 3 & 308,06 & $\mathrm{a}$ & 291,81 & $\mathrm{a}$ & 292,87 & $\mathrm{a}$ & 293,58 & $\mathrm{a}$ & 302,96 & $\mathrm{a}$ & 283,39 & & 268,16 & $\mathrm{a}$ \\
\hline 9 & 280,91 & $\mathrm{a}$ & 226,67 & $\mathrm{~b}$ & 226,59 & $\mathrm{~b}$ & 227,33 & $\mathrm{a}$ & 243,12 & $\mathrm{a}$ & 247,01 & & 266,97 & $\mathrm{a}$ \\
\hline 5 & 281,81 & $\mathrm{a}$ & 232,31 & $\mathrm{~b}$ & 232,53 & $\mathrm{~b}$ & 228,85 & $\mathrm{a}$ & 243,14 & $\mathrm{a}$ & 248,27 & $\mathrm{a}$ & 265,88 & $\mathrm{a}$ \\
\hline 12 & 267,49 & $\mathrm{a}$ & 209,32 & $\mathrm{~b}$ & 213,86 & $\mathrm{~b}$ & 214,67 & $\mathrm{a}$ & 228,75 & $\mathrm{a}$ & 225,58 & $\mathrm{a}$ & 242,64 & $\mathrm{~b}$ \\
\hline 2 & 274,42 & $\mathrm{a}$ & 256,49 & $\mathrm{a}$ & 253,21 & $\mathrm{a}$ & 250,08 & $\mathrm{a}$ & 258,91 & $\mathrm{a}$ & 236,78 & & 240,52 & $\mathrm{~b}$ \\
\hline 4 & 256,14 & $\mathrm{a}$ & 224,49 & $\mathrm{~b}$ & 224,92 & $\mathrm{~b}$ & 230,15 & $\mathrm{a}$ & 234,57 & $\mathrm{a}$ & 223,31 & $\mathrm{~d}$ & 237,38 & $\mathrm{~b}$ \\
\hline 8 & 286,94 & $\mathrm{a}$ & 229,35 & $\mathrm{~b}$ & 232,01 & $\mathrm{~b}$ & 235,65 & $\mathrm{a}$ & 249,19 & $\mathrm{a}$ & 228,48 & & 232,48 & $\mathrm{~b}$ \\
\hline 7 & 276,62 & $\mathrm{a}$ & 243,36 & $\mathrm{~b}$ & 241,79 & $\mathrm{~b}$ & 238,93 & $\mathrm{a}$ & 244,65 & $\mathrm{a}$ & 239,98 & $a$ & 229,26 & $\mathrm{~b}$ \\
\hline 6 & 255,88 & $\mathrm{a}$ & 206,62 & $\mathrm{~b}$ & 210,18 & $\mathrm{~b}$ & 208,58 & $\mathrm{a}$ & 217,01 & $\mathrm{a}$ & 207,41 & $\mathrm{a}$ & 221,91 & $\mathrm{~b}$ \\
\hline 11 & 263,87 & $\mathrm{a}$ & 216,41 & $\mathrm{~b}$ & 218,94 & b & 201,67 & $\mathrm{a}$ & 206,64 & $\mathrm{a}$ & 201,56 & $\mathrm{a}$ & 218,88 & $\mathrm{~b}$ \\
\hline 10 & 282,77 & $\mathrm{a}$ & 209,02 & $\mathrm{~b}$ & 210,56 & $\mathrm{~b}$ & 209,92 & $\mathrm{a}$ & 220,91 & $\mathrm{a}$ & 207,92 & $\mathrm{a}$ & 204,69 & $\mathrm{~b}$ \\
\hline Méd & 277,262 & & 234,26 & & 235,03 & & 232,59 & & 242,8 & & 234,89 & & 243,01 & \\
\hline
\end{tabular}

O ingresso total ficou assim distribuído por tratamentos: leves: T1 - 1,45\%.ano ${ }^{-1}$, T2 1,74\%.ano ${ }^{-1}$, T3 - 1,78\%.ano ${ }^{-1}$ e T4 - 1,76\%.ano ${ }^{-1}$ médios: T5 - 2,00\%.ano ${ }^{-1}$, T6 - 2,33\%.ano ${ }^{-1}$, T7 2,01\%.ano ${ }^{-1}$ e T8 - 2,24\%.ano ${ }^{-1}$; pesados: T9 - 2,17\%.ano ${ }^{-1}$, T10 - 2,28\%.ano ${ }^{-1}$, T11 - 2,56\%.ano ${ }^{-1}$ e T12 2,64\% ano $^{-1}$, com Média Global de 2,08\% ano $^{-1}$, após vinte anos de monitoramento.

Os valores obtidos, para os tratamentos menos intensos são similares aos encontrados por Lieberman; Lieberman (1987) para uma floresta primária na Costa Rica $\left(1,8 \%\right.$.ano $\left.{ }^{-1}\right)$ e por Gomide (1997) nas parcelas sem intervenção na floresta da Jari $\left(1,5 \%\right.$.ano $\left.{ }^{-1}\right)$. Os valores obtidos das intensidades médias e pesadas se assemelham aos obtidos por Silva (2004) em Paragominas, sete anos após a exploração $\left(2,3 \%\right.$.ano ${ }^{-1}$ a $2,5 \%$. ano $\left.^{-1}\right)$.

As taxas de ingressos ficaram abaixo dos valores encontradas por Higuchi et al. (1997) em áreas exploradas e submetidas a desbastes na intensidade de $25 \%$ a $75 \%$ de redução da área basal na região de Manaus (2,9\%.ano ${ }^{-1}$ a 4,2\%.ano $\left.{ }^{-1}\right)$ e por Silva et al. (1995), 11 anos após uma exploração pesada (75 
$\left.\mathrm{m}^{3} \cdot h \mathrm{~h}^{-1}\right)$, porém sem intervenção posterior, na FLONA do Tapajós $\left(3,1 \% \cdot\right.$ ano $\left.^{-1}\right)$. Valores mais altos foram encontrados por Oliveira (2005), também na FLONA do Tapajós, após vinte e dois anos de acompanhamento: em torno de $3,5 \%$.ano ${ }^{-1}$ para os tratamentos mais intensos, em torno de $2,0 \%$.ano ${ }^{-1}$ para os tratamentos menos intensos e de $1,2 \%$.ano ${ }^{-1}$ para a floresta-testemunha.

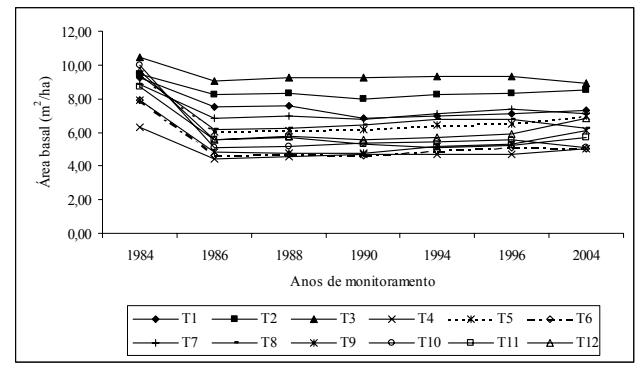

Área Basal $\left(\mathrm{m}^{2} \cdot \mathrm{ha}^{-1}\right)$

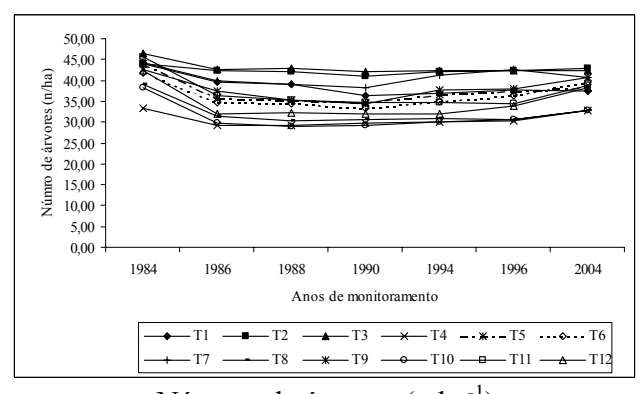

Número de árvores (n.ha $\left.{ }^{-1}\right)$

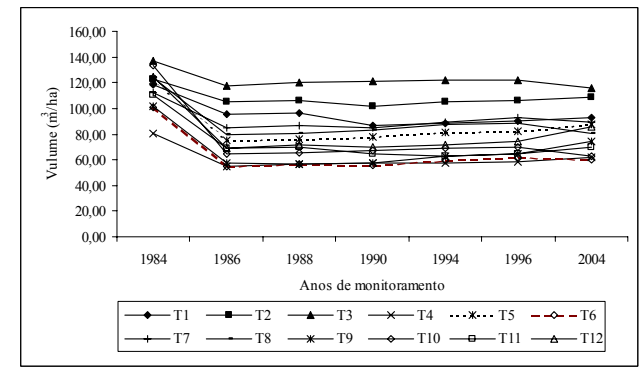

Volume $\left(\mathrm{m}^{3} \cdot \mathrm{ha}^{-1}\right)$

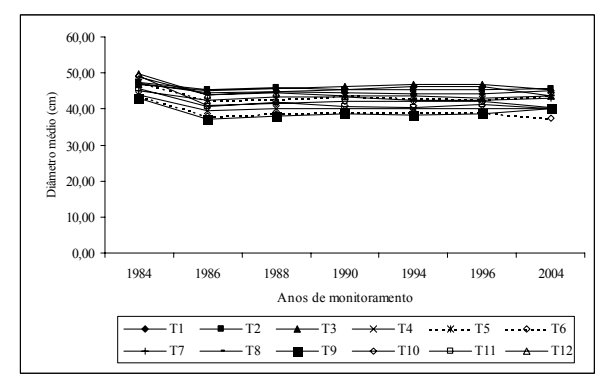

Diâmetro médio $(\mathrm{cm})$

Figura 2. Desenvolvimento da área basal, volume, número de árvores e diâmetro médio das espécies comerciais por tratamento e a cada período de medição na floresta da Jari.

Figure 2. Development of the basal area, volume, number of trees and mean diameter of the commercial species per treatment and at each period of measurement in the forest of the Jari.

$\mathrm{O}$ ingresso das árvores comerciais ficou assim distribuído: tratamentos leves: $\mathrm{T} 1-0,23 \%$.ano ${ }^{-1}$, $\mathrm{T} 2-0,44 \% \cdot \mathrm{ano}^{-1}$, T3 - 0,29\%.ano ${ }^{-1}$ e T4 - 0,52\%.ano ${ }^{-1}$; tratamentos médios: T5 - 0,55\%.ano ${ }^{-1}$, T6 $0,53 \% \cdot$ ano $^{-1}, \mathrm{~T} 7-0,56 \% \cdot \mathrm{ano}^{-1}$ e T8 $-0,36 \%$.ano ${ }^{-1}$; tratamentos pesados: T9 - 0,59\%.ano ${ }^{-1}$, T10 0,48\%.ano ${ }^{-1}$, T11 - 0,59\%.ano ${ }^{-1}$ e T12 - 0,78\%.ano ${ }^{-1}$, com Média Global de 0,49\%.ano ${ }^{-1}$, após vinte anos de monitoramento. Fica evidente que o ingresso de espécies comerciais não foi favorecido pela extração e nem pela aplicação dos tratamentos silviculturais.

A média anual de mortalidade, nos vinte anos de acompanhamento, ficou em torno de $2,15 \%$. ano ${ }^{-1}$ para os tratamentos considerados leves, de aproximadamente $2,74 \%$.ano ${ }^{-1}$ para os médios e de 2,60\% ano ${ }^{-1}$ para os pesados. As maiores taxas de mortalidade foram observadas no período logo após a exploração (1984-1986), com valores em torno de $3,71 \%$. ano $^{-1}$. Essa taxa um pouco elevada se deveu, além da mortalidade natural, à mortalidade ocasionada pela extração das árvores comerciais e pelos danos às árvores remanescentes em conseqüência da derrubada e do arraste das tiras (OLIVEIRA, 2005; LOPES, 1993). A mortalidade foi reduzida nos anos seguintes à exploração, atingindo a taxa de $1,20 \%$.ano ${ }^{-1}$, nove a cinco anos após a exploração (1990-1994). Os tratamentos silviculturais também provocaram um novo aumento nas taxas de mortalidade no período 1996-1994, porém foi observada a mesma tendência de retorno aos níveis encontrados na área antes da aplicação dos tratamentos, com a taxa de 1,50\%.ano ${ }^{-1}$. Declínio acentuado nas taxas de mortalidade foi observado nos trabalhos de Oliveira (2005), Silva (1989) e Higuchi et al. (1997) em florestas manejadas, contudo, essas taxas declinam para valores normais após 10 anos (JONKERS, 1987; De GRAAF, 1986).

Diferenças significativas nas taxas de mortalidade foram observadas no período logo após a colheita de madeira, estando incluídas as árvores que morreram em conseqüência dos danos ocasionados 
pelas atividades de derrubada e arraste das toras, mas não as árvores extraídas (Tabela 4). Os tratamentos leves, T1, T2 e T3, exceto o T4, apresentaram as menores taxas de mortalidade, com as médias dos tratamentos estatisticamente diferentes dos demais tratamentos, que não apresentaram diferenças estatísticas significativas entre si.

As maiores taxas de mortalidade foram observadas no período 1996-1994, logo após a aplicação dos tratamentos silviculturais, contudo, as diferenças entre as médias dos tratamentos, nesse período, não foram estatisticamente significativas (Tabela 4). Esse aumento ocorreu devido à inclusão das árvores aneladas nos cálculos das taxas de mortalidade.

Tabela 4. Efeito das intervenções na mortalidade de árvores, em cada tratamento e a cada período de medição (Taxas de mortalidade média seguidas da mesma letra, para cada ano, não diferem entre si ao nível de 5\% pelo teste de Scott-Knott, na Floresta da Jari).

Table 4. Effect of the interventions on the mortality of trees, for each treatment and for each period of measurement (Rate of mean mortality followed of the same letter, for each year, does not differ between itself at the level of 5\% by the test of Scott-Knott, in the Forest of the Jari).

\begin{tabular}{|c|c|c|c|c|c|c|c|c|c|c|c|c|}
\hline \multirow{3}{*}{$\begin{array}{l}\text { Tratamento } \\
7\end{array}$} & \multicolumn{12}{|c|}{ Mortalidade (\%) } \\
\hline & \multicolumn{2}{|c|}{1986} & \multicolumn{2}{|c|}{1988} & \multicolumn{2}{|c|}{1990} & \multicolumn{2}{|c|}{1994} & \multicolumn{2}{|c|}{1996} & \multicolumn{2}{|c|}{2004} \\
\hline & 3,04 & $\mathrm{a}$ & 2,33 & $\mathrm{a}$ & 1,64 & $\mathrm{a}$ & 1,48 & $\mathrm{a}$ & 4,94 & $\mathrm{a}$ & 2,47 & $\mathrm{a}$ \\
\hline 10 & 4,96 & $\mathrm{a}$ & 1,89 & $\mathrm{a}$ & 1,68 & $\mathrm{a}$ & 1,14 & $\mathrm{a}$ & 7,07 & $\mathrm{a}$ & 2,16 & $\mathrm{a}$ \\
\hline 3 & 1,10 & $\mathrm{~b}$ & 1,60 & $\mathrm{a}$ & 1,78 & $\mathrm{a}$ & 0,93 & $\mathrm{a}$ & 6,10 & $\mathrm{a}$ & 2,07 & $\mathrm{a}$ \\
\hline 8 & 5,75 & $\mathrm{a}$ & 2,06 & $\mathrm{a}$ & 1,65 & $\mathrm{a}$ & 0,94 & $\mathrm{a}$ & 8,67 & $\mathrm{a}$ & 2,04 & $\mathrm{a}$ \\
\hline 6 & 3,80 & $\mathrm{a}$ & 1,76 & $\mathrm{a}$ & 2,42 & $\mathrm{a}$ & 1,26 & $\mathrm{a}$ & 6,04 & $\mathrm{a}$ & 1,49 & $\mathrm{~b}$ \\
\hline 9 & 3,98 & $\mathrm{a}$ & 2,06 & $\mathrm{a}$ & 2,14 & $\mathrm{a}$ & 0,87 & $\mathrm{a}$ & 1,44 & $\mathrm{a}$ & 1,32 & $\mathrm{~b}$ \\
\hline 12 & 5,89 & $\mathrm{a}$ & 1,71 & $\mathrm{a}$ & 1,85 & $\mathrm{a}$ & 1,68 & $\mathrm{a}$ & 3,44 & $\mathrm{a}$ & 1,16 & $\mathrm{~b}$ \\
\hline 4 & 3,84 & $\mathrm{a}$ & 1,25 & $\mathrm{a}$ & 1,79 & $\mathrm{a}$ & 1,24 & $\mathrm{a}$ & 3,97 & $\mathrm{a}$ & 1,13 & $\mathrm{~b}$ \\
\hline 2 & 1,24 & $\mathrm{~b}$ & 1,42 & $\mathrm{a}$ & 1,86 & $\mathrm{a}$ & 1,52 & $\mathrm{a}$ & 7,48 & $\mathrm{a}$ & 1,12 & $\mathrm{~b}$ \\
\hline 5 & 4,16 & $\mathrm{a}$ & 2,27 & $\mathrm{a}$ & 2,15 & $\mathrm{a}$ & 1,00 & $\mathrm{a}$ & 1,31 & $\mathrm{a}$ & 1,06 & $\mathrm{~b}$ \\
\hline 1 & 2,47 & $\mathrm{~b}$ & 1,88 & $\mathrm{a}$ & 3,14 & $\mathrm{a}$ & 0,88 & $\mathrm{a}$ & 0,82 & $\mathrm{a}$ & 0,98 & $\mathrm{~b}$ \\
\hline 11 & 4,32 & $\mathrm{a}$ & 1,57 & $\mathrm{a}$ & 4,02 & $\mathrm{a}$ & 1,41 & $\mathrm{a}$ & 3,61 & $\mathrm{a}$ & 0,96 & $\mathrm{~b}$ \\
\hline Média & \multicolumn{2}{|c|}{3,71} & \multicolumn{2}{|c|}{1,82} & \multicolumn{2}{|c|}{2,18} & \multicolumn{2}{|c|}{1,20} & \multicolumn{2}{|c|}{4,57} & \multicolumn{2}{|c|}{1,50} \\
\hline
\end{tabular}

No último período de acompanhamento (2004-1996), o teste de Scott-Knott discriminou os tratamentos em dois grupos, com o grupo formado pelos tratamentos T7, T10, T3 e T8 apresentando médias superiores às dos demais tratamentos.

As taxas de mortalidade para o período de 1996-1994, sob tratamento silvicultural (refinamento/liberação), são mais altas que em outros períodos no mesmo tratamento. Desconsiderando o caso citado, todas as taxas anuais de mortalidade observadas estão variando de 1,0 a aproximadamente $5,0 \%$, considerado por Alder (1995) como um valor normal, esperado para as florestas tropicais com e sem interferências.

O povoamento comercial apresentou as maiores taxas de mortalidade no período logo após a exploração (1986-1984), com valor médio de 1,17\%.ano ${ }^{-1}$. Essa taxa foi ocasionada pela mortalidade natural e pelos danos às árvores remanescentes, devidos à derrubada e ao arraste das toras, uma vez que a extração das árvores comerciais não foi contada para a obtenção desses valores. Observou-se diferença estatística entre as médias dos tratamentos, com o teste de Scott-Knott discriminando os tratamentos em três grupos. Os tratamentos de intensidade leve apresentaram as menores médias, com destaque para o tratamento T2, que apresentou mortalidade nula (Tabela 5).

A mortalidade foi reduzida nos anos seguintes à exploração para taxas 0,33\%.ano-1 (1988-1986), 0,68\%.ano ${ }^{-1}$ (1990-1988), 0,28\%.ano ${ }^{-1}$ (1994-1990), 0,28\%.ano ${ }^{-1}(1996-1994)$ e 0,35\%.ano ${ }^{-1}$ (20041996), sendo que no intervalo 1990-1988 o grupo formado pelos tratamentos T3, T4, T10 e T8 apresentaram as menores médias, estatisticamente iguais entre si e diferentes das médias dos demais. No povoamento comercial, mais de $50 \%$ dos tratamentos apresentaram balaço negativo ou nulo.

As variações do IPA $\mathrm{DAP}_{\mathrm{D}}, \mathrm{IPA}_{\mathrm{G}}$ e IPA $\mathrm{IP}_{\mathrm{v}}$ foram significativamente diferentes em função do tempo $(\mathrm{p}<0,0001)$. As interações tempo*tratamento, $\mathrm{p}=0,2689, \mathrm{p}=0,0296$ e $\mathrm{p}=0,0475$, respectivamente, demonstram que os $\mathrm{IPA}_{\mathrm{G}}$ e IPA $\mathrm{IP}_{\mathrm{v}}$ variaram significativamente em função dos tratamentos. 
Tabela 5. Efeito das intervenções na mortalidade de árvores comerciais, em cada tratamento e a cada período de medição (Taxas de mortalidade média seguida da mesma letra, para cada ano, não diferem entre si ao nível de 5\% pelo teste de Scott-Knott, na Floresta da Jari).

Table 5. Effect of the interventions on the mortality of commercial trees, for each treatment and at each period of measurement (Rate of mean mortality followed of the same letter, for each year, does not differ between itself to the level of 5\% for the test of Scott-Knott, in the Forest of the Jari).

\begin{tabular}{|c|c|c|c|c|c|c|c|c|c|c|c|c|}
\hline \multirow{3}{*}{$\begin{array}{l}\text { Tratamento } \\
12\end{array}$} & \multicolumn{12}{|c|}{ Mortalidade (\%) } \\
\hline & \multicolumn{2}{|c|}{1986} & \multicolumn{2}{|c|}{1988} & \multicolumn{2}{|c|}{1990} & \multicolumn{2}{|c|}{1994} & \multicolumn{2}{|c|}{1996} & \multicolumn{2}{|c|}{2004} \\
\hline & 2,18 & $\mathrm{a}$ & 0,32 & $\mathrm{a}$ & 1,38 & $\mathrm{a}$ & 0,87 & $\bar{a}$ & 0,00 & $\mathrm{a}$ & 0,08 & $\mathrm{a}$ \\
\hline 5 & 2,08 & $\mathrm{a}$ & 0,52 & $\mathrm{a}$ & 0,66 & $\mathrm{a}$ & 0,30 & $\mathrm{a}$ & 0,16 & $\mathrm{a}$ & 0,40 & $\mathrm{a}$ \\
\hline 11 & 1,79 & $\mathrm{a}$ & 0,42 & $\mathrm{a}$ & 1,37 & $\mathrm{a}$ & 0,65 & $\mathrm{a}$ & 0,19 & $\mathrm{a}$ & 0,29 & $\mathrm{a}$ \\
\hline 10 & 1,76 & $\mathrm{a}$ & 0,47 & $\mathrm{a}$ & 0,05 & $\mathrm{~b}$ & 0,29 & $\mathrm{a}$ & 0,05 & $\mathrm{a}$ & 0,41 & $\mathrm{a}$ \\
\hline 9 & 1,37 & $\mathrm{a}$ & 0,38 & $\mathrm{a}$ & 0,61 & $\mathrm{a}$ & 0,27 & $\mathrm{a}$ & 0,23 & $\mathrm{a}$ & 0,22 & $\mathrm{a}$ \\
\hline 4 & 1,16 & $\mathrm{a}$ & 0,06 & $\mathrm{a}$ & 0,26 & $\mathrm{~b}$ & 0,16 & a & 0,67 & $\mathrm{a}$ & 0,08 & $\mathrm{a}$ \\
\hline 8 & 1,16 & $\mathrm{a}$ & 0,82 & $\mathrm{a}$ & 0,00 & $\mathrm{~b}$ & 0,03 & $\mathrm{a}$ & 0,85 & $\mathrm{a}$ & 0,51 & $\mathrm{a}$ \\
\hline 6 & 0,96 & $\mathrm{a}$ & 0,18 & $\mathrm{a}$ & 0,73 & $\mathrm{a}$ & 0,23 & $\mathrm{a}$ & 0,24 & $\mathrm{a}$ & 0,31 & $\mathrm{a}$ \\
\hline 1 & 0,66 & $\mathrm{~b}$ & 0,27 & $\mathrm{a}$ & 1,00 & $\mathrm{a}$ & 0,08 & a & 0,04 & $\mathrm{a}$ & 0,39 & $\mathrm{a}$ \\
\hline 7 & 0,54 & $\mathrm{~b}$ & 0,41 & $\mathrm{a}$ & 0,92 & $\mathrm{a}$ & 0,24 & a & 0,12 & $\mathrm{a}$ & 0,66 & $\mathrm{a}$ \\
\hline 3 & 0,37 & $\mathrm{~b}$ & 0,00 & $\mathrm{a}$ & 0,29 & $\mathrm{~b}$ & 0,06 & $\mathrm{a}$ & 0,62 & $\mathrm{a}$ & 0,58 & $\mathrm{a}$ \\
\hline 2 & 0,00 & $\mathrm{c}$ & 0,08 & $\mathrm{a}$ & 0,89 & $\mathrm{a}$ & 0,20 & $\mathrm{a}$ & 0,21 & $\mathrm{a}$ & 0,24 & $\mathrm{a}$ \\
\hline Média & 1,17 & & 0,3 & & 0,6 & & 0,2 & & 0,28 & & 0,3 & \\
\hline
\end{tabular}

Silva et al. (1995) encontraram taxa de crescimento de $0,3 \mathrm{~cm} \cdot \mathrm{ano}^{-1}, 13$ anos após a exploração, na FLONA do Tapajós. De Graaf (1986), no Suriname, encontrou 0,4 cm.ano ${ }^{-1}$, nove anos após a exploração, e Silva (2004) encontrou taxas em torno de $0,5 \mathrm{~cm} \cdot$ ano $^{-1}$ para exploração de impacto reduzido e $0,3 \mathrm{~cm} . \mathrm{ano}^{-1}$ para exploração convencional, em Paragominas(PA), sete anos após a exploração de madeira.

As intensidades de redução da área basal não tiveram efeito sobre as taxas de crescimento, que se mantiveram negativas. Resultados contrastantes foram obtidos por Higuchi et al. (1997), que obtiveram incrementos variando de $0,25 \mathrm{~cm} \cdot \mathrm{ano}^{-1}$ a $0,28 \mathrm{~cm} \cdot \mathrm{ano}^{-1}$ nos tratamentos onde foi realizada a redução da área basal através de anelamento, e em torno $0,15 \mathrm{~cm} \cdot \mathrm{ano}^{-1}$ nas parcelas-testemunha.

$\mathrm{O}$ incremento anual líquido em área basal total diferiu significativamente entre os tratamentos após a exploração (1986-1984), com decremento em todos os tratamentos. Os tratamentos leves apresentaram as maiores médias, estatisticamente iguais entre si e superiores às dos demais (Tabela 6).

$\mathrm{O}$ incremento em volume, por sua vez, apresentou diferença significativa entre os tratamentos após o anelamento (1996-1994), com os tratamentos T5, T1 e T9, que não sofreram intervenções póscolheita, apresentando médias estatisticamente iguais, positivas e superiores às dos demais tratamentos (Tabela 6). Houve decrementos em área basal $\left(-0,3520 \mathrm{~m}^{2} \cdot \mathrm{ha}^{-1} \cdot \mathrm{ano}^{-1}\right)$ e volume $\left(-0,3520 \mathrm{~m}^{3} \cdot \mathrm{ha}^{-1} \cdot \mathrm{ano}^{-1}\right)$.

$\mathrm{O}$ incremento periódico anual líquido em diâmetro, área basal e volume comerciais (Tabela 7) teve comportamento similar ao da floresta, incluindo todas as espécies, resultando decremento para 0 $\operatorname{IPA}_{\text {DAP }}\left(-0,42 \mathrm{~cm} \cdot \mathrm{ano}^{-1}\right), \operatorname{IPA}_{\mathrm{G}}\left(-0,27 \mathrm{~m}^{2} \cdot \mathrm{ha}^{-1} \cdot \mathrm{ano}^{-1}\right)$ e $\operatorname{IPA}_{\mathrm{V}}\left(-3,77 \mathrm{~m}^{3} \cdot \mathrm{ha}^{-1} \cdot \mathrm{ano}^{-1}\right)$.

Na região de Santarém (Tapajós), Silva et al. (1996) observaram o crescimento e a produção numa floresta de terra firme, 7 e 13 anos após a exploração. Nessas áreas, os incrementos periódicos anuais em volume de madeira variaram de $1,6 \mathrm{~m}^{3} \cdot \mathrm{ha}^{-1} \cdot$ ano $^{-1}$ em floresta primária não-explorada até 4,8 $\mathrm{m}^{3}$.ha $\mathrm{hano}^{-1}$ em floresta manejada. Considerando apenas as espécies comerciais manejadas com DAP $\geq$ $50 \mathrm{~cm}$, o incremento foi de $1 \mathrm{~m}^{3} \cdot \mathrm{ha}^{-1} \cdot \mathrm{ano}^{-1}$.

Gomide (1997), analisando a dinâmica de crescimento na Floresta da Jari, concluiu que, na floresta primária, o incremento periódico em volume de todas as espécies, durante o período de 19961985, foi de $-0,39 \mathrm{~m}^{3} \cdot \mathrm{ha}^{-1}$.ano ${ }^{-1}$; enquanto que na floresta secundária o IPA foi de $3,53 \mathrm{~m}^{3} \cdot \mathrm{ha}^{-1}$.ano ${ }^{-1}$.

Higuchi et al. (1997), pesquisando o incremento em área basal e volume das "espécies listadas" (comerciais com DAP acima de $10 \mathrm{~cm}$ ), encontraram os seguintes resultados: para a testemunha, 0,07 $\mathrm{m}^{2} \cdot \mathrm{ha}^{-1}$.ano ${ }^{-1}$ e $0,96 \mathrm{~m}^{3} \cdot \mathrm{ha}^{-1}$. ano ${ }^{-1}$; no tratamento com remoção de $25 \%$ de área basal, $0,16 \mathrm{~m}^{2} \cdot \mathrm{ha}^{-1}$. ano ${ }^{-1} \mathrm{e}$ $2,11 \mathrm{~m}^{3} \cdot \mathrm{ha}^{-1} \cdot$ ano $^{-1}$; com remoção de $50 \%$ de área basal, $0,13 \mathrm{~m}^{2} \cdot \mathrm{ha}^{-1}$.ano ${ }^{-1}$ e $1,71 \mathrm{~m}^{3} \cdot \mathrm{ha}^{-1}$. ano ${ }^{-1}$; e com remoção de $75 \%$ de área basal, $0,10 \mathrm{~m}^{2} \cdot \mathrm{ha}^{-1}$. nno $^{-1}$ e $1,58 \mathrm{~m}^{3} \cdot \mathrm{ha}^{-1}$.ano ${ }^{-1}$. Para as espécies comerciais com $\mathrm{DAP} \geq 50 \mathrm{~cm}$, o incremento foi igual ao de Santarém $\left(1,0 \mathrm{~m}^{3} \cdot \mathrm{ha}^{-1} \cdot \mathrm{ano}^{-1}\right)$. 
Tabela 6. Incremento periódico anual em área basal e volume, por tratamento, a cada período de medição, na floresta da Jari (Médias seguidas da mesma letra, para cada ano, não diferem entre si ao nível de 5\% pelo teste de Scott-Knott).

Table 6. Periodic annual increment in basal area and volume, per treatment, at each period of measurement, in the forest of the Jari (Means followed of the same letter, for each year, do not differ between itself to the level of 5\% for the test of Scott-Knott).

\begin{tabular}{|c|c|c|c|c|c|c|c|c|c|c|c|c|}
\hline \multirow{3}{*}{$\begin{array}{l}\text { Tratamento } \\
3\end{array}$} & \multicolumn{12}{|c|}{ Área Basal $\left(\mathrm{m}^{2}\right.$.ano $\left.{ }^{-1}\right)$} \\
\hline & \multicolumn{2}{|l|}{1986} & \multicolumn{2}{|l|}{1988} & \multicolumn{2}{|l|}{1990} & \multicolumn{2}{|l|}{1994} & \multicolumn{2}{|l|}{1996} & \multicolumn{2}{|l|}{2004} \\
\hline & $-0,7293$ & $\mathrm{a}$ & 0,0206 & $\mathrm{a}$ & 0,0149 & $\mathrm{a}$ & 0,2175 & $\mathrm{a}$ & $-0,8517$ & $\mathrm{a}$ & $-0,1199$ & $\mathrm{a}$ \\
\hline 2 & $-0,7898$ & $\mathrm{a}$ & $-0,1126$ & $\mathrm{a}$ & $-0,0983$ & $\mathrm{a}$ & 0,1708 & $\mathrm{a}$ & $-0,9838$ & $\mathrm{a}$ & 0,0669 & $\mathrm{a}$ \\
\hline 1 & $-1,2862$ & $\mathrm{a}$ & $-0,1090$ & $\mathrm{a}$ & $-0,4084$ & $\mathrm{a}$ & 0,2708 & $\mathrm{a}$ & 0,1815 & $\mathrm{a}$ & 0,1841 & $\mathrm{a}$ \\
\hline 4 & $-1,4788$ & $\mathrm{a}$ & 0,0341 & $\mathrm{a}$ & 0,1714 & $\mathrm{a}$ & 0,0855 & $\mathrm{a}$ & $-0,4862$ & $\mathrm{a}$ & 0,1469 & $\mathrm{a}$ \\
\hline 7 & $-1,5681$ & $\mathrm{a}$ & $-0,0792$ & $\mathrm{a}$ & $-0,0779$ & $\mathrm{a}$ & 0,1277 & $\mathrm{a}$ & $-0,2535$ & $\mathrm{a}$ & $-0,0957$ & $\mathrm{a}$ \\
\hline 11 & $-2,2438$ & $\mathrm{~b}$ & 0,0645 & $\mathrm{a}$ & $-0,6112$ & $\mathrm{a}$ & 0,1195 & $\mathrm{a}$ & $-0,2147$ & $\mathrm{a}$ & 0,2095 & $\mathrm{a}$ \\
\hline 6 & $-2,2772$ & $\mathrm{~b}$ & 0,1281 & $\mathrm{a}$ & $-0,0639$ & $\mathrm{a}$ & 0,1746 & $\mathrm{a}$ & $-0,4470$ & $\mathrm{a}$ & 0,1704 & $\mathrm{a}$ \\
\hline 5 & $-2,2934$ & $\mathrm{~b}$ & $-0,0313$ & $\mathrm{a}$ & $-0,1433$ & $\mathrm{a}$ & 0,3087 & $\mathrm{a}$ & 0,2116 & $\mathrm{a}$ & 0,1919 & $\mathrm{a}$ \\
\hline 9 & $-2,4664$ & $\mathrm{~b}$ & $-0,0302$ & $\mathrm{a}$ & 0,0250 & $\mathrm{a}$ & 0,3426 & $\mathrm{a}$ & 0,1795 & $\mathrm{a}$ & 0,2025 & $\mathrm{a}$ \\
\hline 8 & $-2,7360$ & $\mathrm{~b}$ & 0,0747 & $\mathrm{a}$ & 0,1150 & $\mathrm{a}$ & 0,2858 & $\mathrm{a}$ & $-0,9092$ & $\mathrm{a}$ & 0,0520 & $\mathrm{a}$ \\
\hline 12 & $-2,7443$ & $\mathrm{~b}$ & 0,1531 & $\mathrm{a}$ & 0,0373 & $\mathrm{a}$ & 0,2965 & $\mathrm{a}$ & $-0,1406$ & $\mathrm{a}$ & 0,1920 & $\mathrm{a}$ \\
\hline 10 & $-3,3643$ & $\mathrm{~b}$ & 0,0443 & $\mathrm{a}$ & $-0,0195$ & $\mathrm{a}$ & 0,2481 & $\mathrm{a}$ & $-0,6091$ & $\mathrm{a}$ & 0,0074 & $\mathrm{a}$ \\
\hline Média & $-1,9981$ & & 0,0131 & & $-0,0882$ & & 0,2207 & & $-0,3603$ & & 0,1007 & \\
\hline \multirow{2}{*}{ Tratamento } & \multicolumn{12}{|c|}{ Volume $\left(\mathrm{m}^{3}\right.$.ano $\left.{ }^{-1}\right)$} \\
\hline & \multicolumn{2}{|l|}{1986} & 1988 & & \multicolumn{2}{|l|}{1990} & \multicolumn{2}{|l|}{1994} & \multicolumn{2}{|l|}{1996} & \multicolumn{2}{|l|}{2004} \\
\hline 5 & $-31,2663$ & $\mathrm{a}$ & 0,1086 & $\mathrm{a}$ & $-1,6379$ & $\mathrm{a}$ & 3,6502 & $\mathrm{a}$ & 2,6729 & $\mathrm{a}$ & 2,1338 & $\mathrm{a}$ \\
\hline 1 & $-16,9793$ & $\mathrm{a}$ & $-1,1415$ & $\mathrm{a}$ & $-5,0070$ & a & 3,2488 & $\mathrm{a}$ & 2,4346 & $\mathrm{a}$ & 2,2092 & $\mathrm{a}$ \\
\hline 9 & $-34,2559$ & $\mathrm{a}$ & $-0,0404$ & $\mathrm{a}$ & 0,3289 & $\mathrm{a}$ & 4,0333 & $\mathrm{a}$ & 2,0259 & $\mathrm{a}$ & 2,4052 & $\mathrm{a}$ \\
\hline 12 & $-36,7419$ & $\mathrm{a}$ & 2,1777 & $\mathrm{a}$ & 0,3624 & $\mathrm{a}$ & 3,5946 & $\mathrm{a}$ & $-1,6528$ & $\mathrm{a}$ & 2,0610 & $\mathrm{a}$ \\
\hline 7 & $-21,0042$ & $\mathrm{a}$ & $-0,7520$ & $\mathrm{a}$ & $-1,2716$ & $\mathrm{a}$ & 1,4585 & $\mathrm{a}$ & $-2,4323$ & $\mathrm{a}$ & $-1,2916$ & $\mathrm{a}$ \\
\hline 11 & $-29,9764$ & $\mathrm{a}$ & 1,2124 & $\mathrm{a}$ & $-7,6730$ & $\mathrm{a}$ & 1,2694 & $\mathrm{a}$ & $-2,6535$ & $\mathrm{a}$ & 2,0958 & $\mathrm{a}$ \\
\hline 6 & $-31,1124$ & $\mathrm{a}$ & 1,7102 & $\mathrm{a}$ & $-0,7105$ & $\mathrm{a}$ & 2,1528 & $\mathrm{a}$ & $-5,0122$ & $\mathrm{~b}$ & 1,7465 & $\mathrm{a}$ \\
\hline 4 & $-19,9890$ & $\mathrm{a}$ & 0,2054 & $\mathrm{a}$ & 2,3244 & $\mathrm{a}$ & 1,1296 & $\mathrm{a}$ & $-5,8766$ & $\mathrm{~b}$ & 1,7003 & $\mathrm{a}$ \\
\hline 10 & $-46,5763$ & $\mathrm{a}$ & 0,7403 & $\mathrm{a}$ & $-0,2841$ & $\mathrm{a}$ & 2,8048 & $\mathrm{a}$ & $-6,7785$ & $b$ & $-0,3944$ & $\mathrm{a}$ \\
\hline 3 & $-10,2654$ & $\mathrm{a}$ & 0,5071 & $\mathrm{a}$ & 0,3176 & $\mathrm{a}$ & 2,3954 & $\mathrm{a}$ & $-10,2139$ & $\mathrm{~b}$ & $-1,8404$ & $\mathrm{a}$ \\
\hline 8 & $-36,3748$ & $\mathrm{a}$ & 1,2760 & $\mathrm{a}$ & 1,6204 & $\mathrm{a}$ & 3,4555 & $\mathrm{a}$ & $-10,8042$ & $\mathrm{~b}$ & 0,4791 & $\mathrm{a}$ \\
\hline 2 & $-11,3234$ & $\mathrm{a}$ & $-1,5741$ & $\mathrm{a}$ & $-1,3948$ & $\mathrm{a}$ & 2,2567 & $\mathrm{a}$ & $-11,5468$ & $\mathrm{~b}$ & 0,4524 & $\mathrm{a}$ \\
\hline Média & $-27,155$ & & 0,3691 & & $-1,0854$ & & 2,6208 & & $-4,1531$ & & 0,9797 & \\
\hline
\end{tabular}

Segundo De Graaf (1999), o incremento em área basal nas áreas manejadas com uso de tratamentos silviculturais foi de $0,6 \mathrm{~m}^{2} \cdot \mathrm{ha}^{-1}$. Nos primeiros 14 anos após a exploração seletiva de madeira, o incremento periódico anual em volume das espécies comerciais variou de 2 a $4,5 \mathrm{~m}^{3} \cdot \mathrm{ha}^{-1}$.ano ${ }^{-1}$, em função da lista de espécies, principalmente para as primeiras classes de diâmetro.

\section{Povoamento total e comercial bruto}

A análise do crescimento bruto, isto é, desconsiderando-se tanto o recrutamento quanto a mortalidade, para as variáveis área basal, volume, diâmetro médio e respectivos incrementos periódicos anuais, para o período total de estudo, foi realizada devido à forte influência que, principalmente, a mortalidade tem sobre a produtividade. Ao morrer uma árvore de grande porte, a substituição da biomassa perdida é lenta, havendo inclusive decrementos em muitos casos.

A área basal de todas as árvores do povoamento, incluindo todas as espécies que se mantêm vivas desde a primeira medição, apresentou as maiores médias para os tratamentos leves em conjunto com os tratamentos que não sofreram interferência pós-exploratória. Os grupos formados pelo teste de Scott-Knott antes da exploração mantiveram-se para todo o período estudado. Já para o volume, o teste de Scott-Knott, a princípio, classificou os tratamentos em um único grupo. A partir de 1988, houve a discriminação em dois grupos, que se mantiveram até a última avaliação, com os tratamentos T4 e T2 
mudando de grupo com a aplicação dos tratamentos silviculturais. O diâmetro médio das árvores remanescentes não foi favorecido pela extração e nem pelo desbaste. Pode-se observar que a exploração madeireira aliada ao tratamento silvicultural teve efeito positivo sobre o crescimento em área basal. No entanto, os tratamentos submetidos à exploração de baixa intensidade (T1, T2, T3 e T4) e os tratamentos que não receberam anelamento após a colheita (T1, T5 e T9) apresentaram as maiores médias, resultado este diferente do esperado (Tabela 8).

Tabela 7. Incremento periódico anual em diâmetro, área basal e volume por tratamento para os anos de monitoramento, para o povoamento comercial líquido (Médias seguidas da mesma letra, em cada ano, não diferem entre si ao nível de 5\% pelo teste de Scott-Knott).

Table 7. Periodic annual increment in diameter, basal area and volume per treatment for the years of monitoring, for the net commercial stand (Means followed same letter, in each year, do not differ between itself to the level of $5 \%$ for the test of Scott-Knott).

\begin{tabular}{|c|c|c|c|c|c|c|c|c|c|c|c|c|}
\hline \multirow{2}{*}{ Tratamento } & \multicolumn{12}{|c|}{ Incremento periódico anual em diâmetro - IPA DAP $\left(\mathrm{cm}^{-a n 0^{-1}}\right)$} \\
\hline & 1986 & & 1988 & & 1990 & & 1994 & & 1996 & & 2004 & \\
\hline 1 & $-1,6882$ & a & 0,2210 & a & $-0,1438$ & a & $-0,0981$ & $\mathrm{a}$ & 0,0416 & $\mathrm{a}$ & 0,1028 & $\mathrm{a}$ \\
\hline 2 & $-1,2209$ & a & 0,2591 & a & $-0,2060$ & a & $-0,0146$ & a & $-0,0281$ & $\mathrm{a}$ & 0,0453 & $\mathrm{a}$ \\
\hline 3 & $-0,9805$ & a & 0,2274 & a & 0,3296 & a & 0,0926 & a & $-0,0201$ & $\mathrm{a}$ & $-0,1571$ & a \\
\hline 4 & $-2,8454$ & a & 0,2763 & a & 0,0887 & a & 0,0047 & a & $-0,1131$ & $\mathrm{a}$ & 0,0178 & a \\
\hline 5 & $-3,1073$ & $\mathrm{a}$ & 0,1478 & $\mathrm{a}$ & 0,4332 & $\mathrm{a}$ & $-0,1584$ & $\mathrm{a}$ & $-0,1295$ & $\mathrm{a}$ & 0,0802 & $\mathrm{a}$ \\
\hline 6 & $-3,5387$ & $\mathrm{a}$ & 0,2852 & $\mathrm{a}$ & 0,1176 & $\mathrm{a}$ & 0,0195 & $\mathrm{a}$ & 0,0004 & $\mathrm{a}$ & $-0,1722$ & $\mathrm{a}$ \\
\hline 7 & $-1,7539$ & $\mathrm{a}$ & 0,5184 & $\mathrm{a}$ & $-0,0810$ & $\mathrm{a}$ & $-0,1806$ & $\mathrm{a}$ & $-0,0981$ & $\mathrm{a}$ & 0,0909 & $\mathrm{a}$ \\
\hline 8 & $-3,1523$ & $\mathrm{a}$ & 0,4196 & $\mathrm{a}$ & 0,2926 & $\mathrm{a}$ & 0,2463 & $\mathrm{a}$ & 0,0178 & $\mathrm{a}$ & $-0,3436$ & $\mathrm{a}$ \\
\hline 9 & $-3,7737$ & $\mathrm{a}$ & 0,3957 & $\mathrm{a}$ & 0,2908 & $\mathrm{a}$ & $-0,0500$ & $\mathrm{a}$ & 0,1901 & $\mathrm{a}$ & 0,1777 & $\mathrm{a}$ \\
\hline 10 & $-5,1923$ & $\mathrm{a}$ & 0,3078 & $\mathrm{a}$ & 0,3158 & $\mathrm{a}$ & $-0,0315$ & $\mathrm{a}$ & 0,1045 & $\mathrm{a}$ & $-0,2132$ & $\mathrm{a}$ \\
\hline 11 & $-3,0236$ & $\mathrm{a}$ & 0,4459 & $\mathrm{a}$ & $-0,5113$ & $\mathrm{a}$ & $-0,0328$ & $\mathrm{a}$ & 0,3752 & $\mathrm{a}$ & $-0,1211$ & $\mathrm{a}$ \\
\hline 12 & $-3,5359$ & $\mathrm{a}$ & 0,2804 & $\mathrm{a}$ & $-0,5128$ & $\mathrm{a}$ & $-0,0027$ & $\mathrm{a}$ & $-0,2917$ & $\mathrm{a}$ & 0,0898 & $\mathrm{a}$ \\
\hline Média & $-2,8177$ & & 0,3154 & & 0,0345 & & $-0,0171$ & & 0,0041 & & $-0,0335$ & \\
\hline \multirow{2}{*}{ Tratamento } & \multicolumn{11}{|c|}{ Incremento periódico anual em área basal - $\operatorname{IPA}_{\mathrm{G}}\left(\mathrm{m}^{2}\right.$.ano-1 $)$} & \\
\hline & 1986 & & 1988 & & 1990 & & 1994 & & 1996 & & 2004 & \\
\hline 2 & $-0,7741$ & $\mathrm{a}$ & 0,0466 & $\mathrm{a}$ & $-0,1509$ & $\mathrm{~b}$ & 0,0648 & $\mathrm{a}$ & 0,0285 & $\mathrm{a}$ & 0,0212 & $\mathrm{a}$ \\
\hline 3 & $-0,9045$ & $\mathrm{a}$ & 0,0955 & $\mathrm{a}$ & 0,0135 & $\mathrm{a}$ & 0,0224 & $\mathrm{a}$ & $-0,0039$ & $\mathrm{a}$ & $-0,0539$ & $\mathrm{a}$ \\
\hline 1 & $-1,1097$ & $\mathrm{a}$ & 0,0303 & $\mathrm{a}$ & $-0,3217$ & $\mathrm{~b}$ & 0,0342 & $\mathrm{a}$ & 0,0694 & $\mathrm{a}$ & 0,0251 & $\mathrm{a}$ \\
\hline 4 & $-1,1903$ & $\mathrm{a}$ & 0,0587 & $\mathrm{a}$ & 0,0435 & $\mathrm{a}$ & $-0,0006$ & $\mathrm{a}$ & 0,0238 & $\mathrm{a}$ & 0,0389 & $\mathrm{a}$ \\
\hline 7 & $-1,2617$ & $\mathrm{a}$ & 0,0592 & $\mathrm{a}$ & $-0,0654$ & $\mathrm{a}$ & 0,0857 & $\mathrm{a}$ & 0,1302 & $\mathrm{a}$ & $-0,0314$ & $\mathrm{a}$ \\
\hline 9 & $-1,9504$ & $\mathrm{~b}$ & $-0,0355$ & $\mathrm{a}$ & 0,0062 & $\mathrm{a}$ & 0,1128 & $\mathrm{a}$ & 0,0695 & $\mathrm{a}$ & 0,0929 & $\mathrm{a}$ \\
\hline 11 & $-1,9936$ & $\mathrm{~b}$ & 0,0432 & $\mathrm{a}$ & $-0,1746$ & $\mathrm{~b}$ & $-0,0401$ & $\mathrm{a}$ & 0,0717 & $\mathrm{a}$ & 0,0548 & $\mathrm{a}$ \\
\hline 8 & $-2,0278$ & $\mathrm{~b}$ & 0,0136 & $\mathrm{a}$ & 0,1002 & $\mathrm{a}$ & 0,0784 & $\mathrm{a}$ & 0,0103 & $\mathrm{a}$ & $-0,0637$ & $\mathrm{a}$ \\
\hline 6 & $-2,0794$ & $\mathrm{~b}$ & 0,0496 & $\mathrm{a}$ & $-0,0298$ & $\mathrm{a}$ & 0,0709 & a & 0,0988 & $\mathrm{a}$ & $-0,0065$ & $\mathrm{a}$ \\
\hline 5 & $-2,1427$ & $\mathrm{~b}$ & 0,0222 & $\mathrm{a}$ & 0,0507 & $\mathrm{a}$ & 0,0629 & $\mathrm{a}$ & 0,0482 & $\mathrm{a}$ & 0,0503 & $\mathrm{a}$ \\
\hline 12 & $-2,5811$ & $\mathrm{~b}$ & 0,1006 & $\mathrm{a}$ & $-0,0809$ & $\mathrm{a}$ & 0,0249 & $\mathrm{a}$ & 03 & $\mathrm{a}$ & 0,1067 & $\mathrm{a}$ \\
\hline 10 & $-3,0662$ & $\mathrm{~b}$ & 0,0389 & $\mathrm{a}$ & 0,0737 & $\mathrm{a}$ & 0,0215 & $\mathrm{a}$ & 0,0561 & $\mathrm{a}$ & $-0,0536$ & $\mathrm{a}$ \\
\hline Média & $-1,7568$ & & 0,0436 & & $-0,0446$ & & 0,0448 & & 0,0611 & & 0,0151 & \\
\hline \multirow{2}{*}{ Tratamento } & \multicolumn{12}{|c|}{ Incremento periódico anual em volume - $\operatorname{IPA}_{\mathrm{Y}}\left(\mathrm{m}^{3} . \mathrm{ano}^{-1}\right)$} \\
\hline & 1986 & & 1988 & & 1990 & & 1994 & & 1996 & & 2004 & \\
\hline 2 & $-11,0293$ & $\mathrm{a}$ & 0,6610 & $\mathrm{a}$ & $-2,0664$ & $\mathrm{~b}$ & 0,9089 & $\mathrm{a}$ & 0,4841 & $\mathrm{a}$ & 0,2821 & $\mathrm{a}$ \\
\hline 3 & $-12,3374$ & $\mathrm{a}$ & 1,2668 & $\mathrm{a}$ & 0,2893 & $\mathrm{a}$ & 0,2095 & $\mathrm{a}$ & 0,0596 & $\mathrm{a}$ & $-0,8013$ & $\mathrm{a}$ \\
\hline 1 & $-14,9118$ & $\mathrm{a}$ & 0,5467 & $\mathrm{a}$ & $-4,3221$ & $\mathrm{~b}$ & 0,4223 & $\mathrm{a}$ & 0,8875 & $\mathrm{a}$ & 0,3695 & $\mathrm{a}$ \\
\hline 4 & $-16,3621$ & $\mathrm{a}$ & 0,8103 & $\mathrm{a}$ & 0,5441 & $\mathrm{a}$ & 0,0135 & $\mathrm{a}$ & 0,3010 & $\mathrm{a}$ & 0,4508 & $\mathrm{a}$ \\
\hline 7 & $-17,4203$ & $\mathrm{a}$ & 0,8886 & $\mathrm{a}$ & $-0,7844$ & $\mathrm{a}$ & 1,0809 & $\mathrm{a}$ & 1,7836 & $\mathrm{a}$ & $-0,3972$ & $\mathrm{a}$ \\
\hline 11 & $-26,7063$ & $\mathrm{~b}$ & 0,6829 & $\mathrm{a}$ & $-2,3621$ & $\mathrm{~b}$ & $-0,5743$ & $\mathrm{a}$ & 0,9557 & $\mathrm{a}$ & 0,6435 & $\mathrm{a}$ \\
\hline 9 & $-27,7578$ & $\mathrm{~b}$ & $-0,3143$ & $\mathrm{a}$ & 0,2828 & $\mathrm{a}$ & 1,3646 & $\mathrm{a}$ & 0,8304 & $\mathrm{a}$ & 1,1919 & $\mathrm{a}$ \\
\hline 8 & $-27,7898$ & $\mathrm{~b}$ & 0,2645 & $\mathrm{a}$ & 1,4049 & $\mathrm{a}$ & 1,1052 & $\mathrm{a}$ & 0,3095 & $\mathrm{a}$ & $-0,9375$ & $\mathrm{a}$ \\
\hline 6 & $-28,9127$ & $\mathrm{~b}$ & 0,8180 & $\mathrm{a}$ & $-0,3351$ & $\mathrm{a}$ & 0,9124 & $\mathrm{a}$ & 1,2455 & $\mathrm{a}$ & $-0,2273$ & $\mathrm{a}$ \\
\hline 5 & $-29,4646$ & $\mathrm{~b}$ & 0,3501 & $\mathrm{a}$ & 0,7493 & $\mathrm{a}$ & 0,7707 & $\mathrm{a}$ & 0,6677 & $\mathrm{a}$ & 0,6893 & $\mathrm{a}$ \\
\hline 12 & $-35,2542$ & $\mathrm{~b}$ & 1,3102 & $\mathrm{a}$ & $-0,9753$ & $\mathrm{a}$ & 0,4134 & a & 1,5391 & $\mathrm{a}$ & 1,3570 & $\mathrm{a}$ \\
\hline 10 & $-43,6168$ & $\mathrm{~b}$ & 0,6363 & $\mathrm{a}$ & 0,9586 & $\mathrm{a}$ & 0,3332 & $\mathrm{a}$ & 0,7001 & $\mathrm{a}$ & $-0,9028$ & $\mathrm{a}$ \\
\hline Média & $-24,2969$ & & 0,6601 & & $-0,5514$ & & 0,5800 & & 0,8137 & & 0,1432 & \\
\hline
\end{tabular}


Tabela 8. Área basal, volume e diâmetro médio por tratamento para os anos de monitoramento, para o povoamento total bruto (Médias seguidas da mesma letra, em cada ano, não diferem entre si ao nível de 5\% pelo teste de Scott-Knott).

Table 8. Basal area, volume and mean diameter per treatment for the years of monitoring, for the gross total stand (Means followed of the same letter, in each year, do not differ between it self to the level of $5 \%$ for the test of Scott-Knott).

\begin{tabular}{|c|c|c|c|c|c|c|c|c|c|c|c|c|c|c|}
\hline \multirow{2}{*}{ Trat. } & \multicolumn{14}{|c|}{ Area basal(m²) } \\
\hline & \multicolumn{2}{|l|}{1984} & \multicolumn{2}{|l|}{1986} & \multicolumn{2}{|l|}{1988} & \multicolumn{2}{|l|}{1990} & \multicolumn{2}{|l|}{1994} & \multicolumn{2}{|l|}{1996} & \multicolumn{2}{|l|}{2004} \\
\hline 1 & 16,8751 & $\mathrm{a}$ & 17,1956 & $\mathrm{a}$ & 17,7246 & $\mathrm{a}$ & 18,2583 & $\mathrm{a}$ & 19,2546 & $\mathrm{a}$ & 19,6497 & $\mathrm{a}$ & 21,3103 & $\mathrm{a}$ \\
\hline 3 & 14,7754 & $\mathrm{a}$ & 15,0798 & $\mathrm{a}$ & 15,4710 & $\mathrm{a}$ & 15,9775 & $\mathrm{a}$ & 16,8727 & $\mathrm{a}$ & 17,2814 & $\mathrm{a}$ & 19,1704 & $\mathrm{a}$ \\
\hline 5 & 14,4523 & $\mathrm{a}$ & 14,7748 & $\mathrm{a}$ & 15,2829 & $\mathrm{a}$ & 15,8513 & $\mathrm{a}$ & 16,7469 & $\mathrm{a}$ & 17,1929 & $\mathrm{a}$ & 18,8695 & $\mathrm{a}$ \\
\hline 9 & 14,2034 & $\mathrm{a}$ & 14,5634 & $\mathrm{a}$ & 15,0864 & $\mathrm{a}$ & 15,6710 & $\mathrm{a}$ & 16,7211 & $\mathrm{a}$ & 17,2288 & $\mathrm{a}$ & 19,0010 & $\mathrm{a}$ \\
\hline 4 & 13,8274 & $\mathrm{a}$ & 14,1291 & $\mathrm{a}$ & 14,5575 & $\mathrm{a}$ & 15,0119 & $\mathrm{a}$ & 15,7220 & $\mathrm{a}$ & 16,0873 & $\mathrm{a}$ & 17,4258 & $\mathrm{a}$ \\
\hline 2 & 13,6839 & $\mathrm{a}$ & 13,9938 & $\mathrm{a}$ & 14,4233 & $\mathrm{a}$ & 14,8893 & $\mathrm{a}$ & 15,7412 & $\mathrm{a}$ & 16,1670 & $\mathrm{a}$ & 17,8035 & $\mathrm{a}$ \\
\hline 8 & 12,5679 & $\mathrm{~b}$ & 12,7809 & $\mathrm{~b}$ & 13,1431 & $\mathrm{~b}$ & 13,5468 & $\mathrm{~b}$ & 14,2122 & $\mathrm{~b}$ & 14,5711 & $\mathrm{~b}$ & 16,0716 & $\mathrm{~b}$ \\
\hline 7 & 12,4241 & $\mathrm{~b}$ & 12,7054 & $\mathrm{~b}$ & 13,1098 & $\mathrm{~b}$ & 13,5935 & $\mathrm{~b}$ & 14,3439 & $\mathrm{~b}$ & 14,7461 & $\mathrm{~b}$ & 16,3432 & $\mathrm{~b}$ \\
\hline 12 & 12,2051 & $\mathrm{~b}$ & 12,5153 & $\mathrm{~b}$ & 12,9927 & $\mathrm{~b}$ & 13,5057 & $\mathrm{~b}$ & 14,4547 & $\mathrm{~b}$ & 14,8782 & $\mathrm{~b}$ & 16,5261 & $\mathrm{~b}$ \\
\hline 6 & 11,5563 & $\mathrm{~b}$ & 11,8615 & $\mathrm{~b}$ & 12,3104 & $\mathrm{~b}$ & 12,7 & $\mathrm{~b}$ & 13,4963 & $\mathrm{~b}$ & 13, & $\mathrm{~b}$ & 15,3051 & $\mathrm{~b}$ \\
\hline 11 & 11,1857 & $\mathrm{~b}$ & 11,4390 & $\mathrm{~b}$ & 11,8750 & $\mathrm{~b}$ & 12,3729 & $\mathrm{~b}$ & 13,1962 & $\mathrm{~b}$ & 13,6050 & $\mathrm{~b}$ & 14,9697 & $\mathrm{~b}$ \\
\hline 10 & 10,6290 & $\mathrm{~b}$ & 10,8596 & $\mathrm{~b}$ & 11,2692 & $\mathrm{~b}$ & 11,6695 & $\mathrm{~b}$ & 12,3624 & $\mathrm{~b}$ & 12,7383 & $\mathrm{~b}$ & 14,1041 & $\mathrm{~b}$ \\
\hline Média & 13,1988 & & 13,4915 & & 13,9372 & & 14,4236 & & 15,2604 & & 15,6685 & & 17,2417 & \\
\hline \multirow{2}{*}{ Trat. } & \multicolumn{14}{|c|}{ Volume $\left(\mathrm{m}^{3}\right)$} \\
\hline & 1984 & & 1986 & & 1988 & & 1990 & & 1994 & & 1996 & & 2004 & \\
\hline 1 & 203,305 & $\mathrm{a}$ & 207,583 & $\mathrm{a}$ & 214,624 & $\mathrm{a}$ & 221,7531 & $\mathrm{a}$ & 234,8609 & $\mathrm{a}$ & 240,3058 & $\mathrm{a}$ & 262,8478 & $\mathrm{a}$ \\
\hline 3 & 180,855 & $\mathrm{a}$ & 184,781 & $\mathrm{a}$ & 189,781 & $\mathrm{a}$ & 196 & $\mathrm{a}$ & 208,2697 & $\mathrm{a}$ & 213 & $\mathrm{a}$ & 093 & $\mathrm{a}$ \\
\hline 5 & 172,086 & $\mathrm{a}$ & 176,338 & $\mathrm{a}$ & 182,977 & $\mathrm{a}$ & 190 & $\mathrm{a}$ & 202 & $\mathrm{a}$ & 208 & $\mathrm{a}$ & 750 & $\mathrm{a}$ \\
\hline 9 & 167,395 & $\mathrm{a}$ & 172,054 & $\mathrm{a}$ & 178,933 & $\mathrm{a}$ & 186 , & $\mathrm{a}$ & 200,7050 & $\mathrm{a}$ & 207, & $\mathrm{a}$ & 424 & $\mathrm{a}$ \\
\hline 4 & 161,737 & $\mathrm{a}$ & 165,697 & $\mathrm{a}$ & 171,171 & $\mathrm{a}$ & 176,9 & $\mathrm{a}$ & 186,4500 & $\mathrm{~b}$ & 191, & $\mathrm{b}$ & 209,0759 & $\mathrm{~b}$ \\
\hline 2 & 161,040 & $\mathrm{a}$ & 165 & $\mathrm{a}$ & 170 & $\mathrm{a}$ & 176 & $\mathrm{a}$ & 188 & $\mathrm{~b}$ & 19 & $\mathrm{~b}$ & 216 , & $\mathrm{b}$ \\
\hline 8 & 152,794 & $\mathrm{a}$ & 155,558 & $\mathrm{a}$ & 160,295 & $\mathrm{~b}$ & 165,6773 & $\mathrm{~b}$ & 174, & $\mathrm{~b}$ & 179 & $\mathrm{~b}$ & 200 & $\mathrm{~b}$ \\
\hline 7 & 147,160 & $\mathrm{a}$ & 150,862 & $\mathrm{a}$ & 156,083 & $\mathrm{~b}$ & 162,4165 & $\mathrm{~b}$ & 172 , & $\mathrm{b}$ & 177 & $\mathrm{~b}$ & 199 & $\mathrm{~b}$ \\
\hline 12 & 144,141 & $\mathrm{a}$ & 148,204 & $\mathrm{a}$ & 154,286 & $\mathrm{~b}$ & 161 , & $\mathrm{b}$ & 173,8275 & $\mathrm{~b}$ & 179,6150 & $\mathrm{~b}$ & 202,0677 & $\mathrm{~b}$ \\
\hline 6 & , 163 & $\mathrm{a}$ & 140,087 & $\mathrm{a}$ & 146,081 & $\mathrm{~b}$ & 151,6 & $\mathrm{~b}$ & 161,7001 & $\mathrm{~b}$ & 166 & $\mathrm{~b}$ & 057 & $\mathrm{~b}$ \\
\hline 11 & 129,219 & $\mathrm{a}$ & 132,432 & $\mathrm{a}$ & 138,134 & $\mathrm{~b}$ & 144,7337 & $\mathrm{~b}$ & 155,8338 & $\mathrm{~b}$ & 161,1461 & $\mathrm{~b}$ & 179,6201 & $\mathrm{~b}$ \\
\hline 10 & 124,501 & $\mathrm{a}$ & 127,494 & $\mathrm{a}$ & 132,815 & $\mathrm{~b}$ & 138,3058 & $\mathrm{~b}$ & 147,5980 & $\mathrm{~b}$ & 152,5459 & $\mathrm{~b}$ & 171,2123 & $\mathrm{~b}$ \\
\hline Média & 156,7001 & & 160,5210 & & 166,3093 & & 172,7240 & & 183,9059 & & 189,3960 & & 210,8546 & \\
\hline \multirow{2}{*}{ Trat. } & \multicolumn{14}{|c|}{ Diâmetro médio(cm) } \\
\hline & 1984 & & 1986 & & 1988 & & 1990 & & 1994 & & 1996 & & 2004 & \\
\hline 1 & 35,77 & $\mathrm{a}$ & 36,18 & $\mathrm{a}$ & 36,80 & $\mathrm{a}$ & 37,46 & $\mathrm{a}$ & 38,63 & $\mathrm{a}$ & 39,07 & $\mathrm{a}$ & 40,87 & $\mathrm{a}$ \\
\hline 2 & 34,68 & $\mathrm{a}$ & 35,13 & $\mathrm{a}$ & 35,79 & $\mathrm{a}$ & 36,48 & $\mathrm{a}$ & 37,64 & $\mathrm{a}$ & 38,19 & $\mathrm{a}$ & 40,28 & $\mathrm{a}$ \\
\hline 3 & 36,83 & $\mathrm{a}$ & 37,32 & $\mathrm{a}$ & 37,94 & $\mathrm{a}$ & & $\mathrm{a}$ & 40,00 & $\mathrm{a}$ & 40,58 & $\mathrm{a}$ & 43,09 & $\mathrm{a}$ \\
\hline 4 & 34,27 & $\mathrm{a}$ & 34,70 & $\mathrm{a}$ & 35,28 & $\mathrm{a}$ & 35,92 & $\mathrm{a}$ & 36,90 & $\mathrm{a}$ & 37,37 & $\mathrm{a}$ & 39,13 & $\mathrm{a}$ \\
\hline 5 & 35,93 & $\mathrm{a}$ & 36,38 & $\mathrm{a}$ & 37,09 & $\mathrm{a}$ & 37,84 & $\mathrm{a}$ & 39,02 & $\mathrm{a}$ & 39,54 & $\mathrm{a}$ & 41,49 & $\mathrm{a}$ \\
\hline 6 & 35,18 & $\mathrm{a}$ & 35,68 & $\mathrm{a}$ & 36,44 & $\mathrm{a}$ & & $\mathrm{a}$ & 38,34 & $\mathrm{a}$ & 38,91 & $\mathrm{a}$ & 40,95 & $\mathrm{a}$ \\
\hline 7 & 35,86 & $\mathrm{a}$ & 36,32 & $\mathrm{a}$ & 37,02 & $\mathrm{a}$ & 37,79 & $\mathrm{a}$ & 38,96 & $\mathrm{a}$ & 39,54 & $\mathrm{a}$ & 41,78 & $\mathrm{a}$ \\
\hline 8 & 36,68 & $\mathrm{a}$ & 37,07 & $\mathrm{a}$ & 37,71 & $\mathrm{a}$ & 38,38 & $\mathrm{a}$ & 39,47 & $\mathrm{a}$ & 40,02 & $\mathrm{a}$ & 42,21 & $\mathrm{a}$ \\
\hline 9 & 34,97 & $\mathrm{a}$ & 35,50 & $\mathrm{a}$ & 36,27 & $\mathrm{a}$ & 37,06 & $\mathrm{a}$ & 38,42 & $\mathrm{a}$ & 39,05 & $\mathrm{a}$ & 41,17 & $\mathrm{a}$ \\
\hline 10 & 34,86 & $\mathrm{a}$ & 35,32 & $\mathrm{a}$ & 36,06 & $\mathrm{a}$ & 36,79 & $\mathrm{a}$ & 37,97 & $\mathrm{a}$ & 38,59 & $\mathrm{a}$ & 40,77 & $\mathrm{a}$ \\
\hline 11 & 34,43 & $\mathrm{a}$ & 34,87 & $\mathrm{a}$ & 35,62 & $\mathrm{a}$ & 36,43 & $\mathrm{a}$ & 37,73 & $\mathrm{a}$ & 38,35 & $\mathrm{a}$ & 40,33 & $\mathrm{a}$ \\
\hline 12 & 35,42 & $\mathrm{a}$ & 35,95 & $\mathrm{a}$ & 36,69 & $\mathrm{a}$ & 37,48 & $\mathrm{a}$ & 38,85 & $\mathrm{a}$ & 39,45 & $\mathrm{a}$ & 41,74 & $\mathrm{a}$ \\
\hline Média & 35,41 & & 35,87 & & 36,56 & & 37,29 & & 38,50 & & 39,06 & & 41,15 & \\
\hline
\end{tabular}

Os incrementos $\operatorname{IPA}_{\text {DAP }}\left(0,30 \mathrm{~cm} \cdot \mathrm{ano}^{-1}\right), \operatorname{IPA}_{\mathrm{G}}\left(0,21 \mathrm{~m}^{2} \cdot \mathrm{ha}^{-1} \cdot \mathrm{ano}^{-1}\right)$ e IPAV $\left(2,73 \mathrm{~m}^{3} \cdot \mathrm{ha}^{-1} \cdot\right.$ ano $\left.^{-1}\right)$ variaram significativamente somente em função do tempo. 
Considerando o povoamento comercial bruto, a área basal, o volume e o diâmetro médio variaram significativamente somente em função do tempo. Na figura 3, ao se observar o desenvolvimento das curvas, verificou-se que as reduções em área basal ocasionadas pela extração aliada ao desbaste não provocaram uma resposta rápida no crescimento das árvores de valor comercial.

Os incrementos $\operatorname{IPA}_{\mathrm{DAP}}\left(0,38 \mathrm{~cm} \cdot \mathrm{ano}^{-1}\right), \operatorname{IPA}_{\mathrm{G}}\left(0,07 \mathrm{~m}^{2} \cdot \mathrm{ha}^{-1} \cdot \mathrm{ano}^{-1}\right)$ e IPA $\mathrm{V}\left(0,95 \mathrm{~m}^{3} \cdot \mathrm{ha}^{-1} \cdot \mathrm{ano}^{-1}\right)$ das árvores comerciais presentes desde a primeira medição variaram significativamente somente em função do tempo.

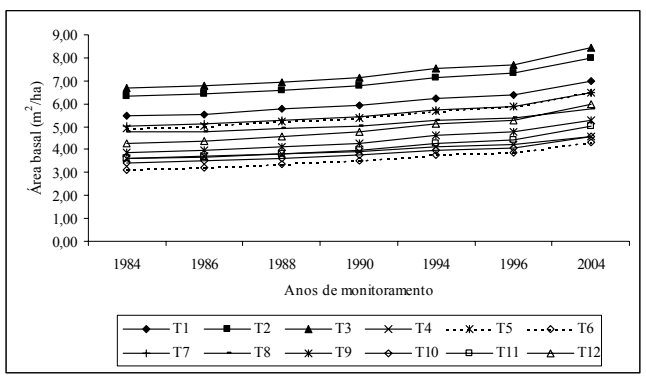

Área basal $\left(\mathrm{m}^{2} \cdot \mathrm{ha}^{-1}\right)$

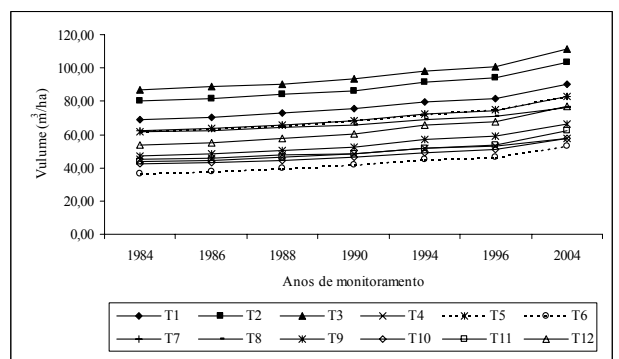

Volume $\left(\mathrm{m}^{3} \cdot \mathrm{ha}^{-1}\right)$

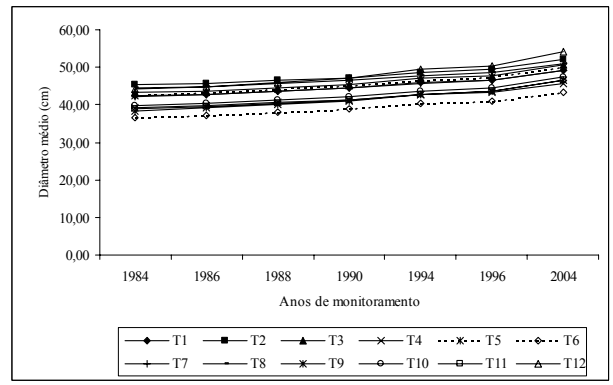

Diâmetro médio (n.ha $\left.{ }^{-1}\right)$

Figura 3. Desenvolvimento da área basal, volume e número de árvores por tratamento e a cada período de medição para o povoamento comercial bruto na floresta da Jari.

Figure 3. Development of the basal area, volume and number of trees by treatment and to each period of measurement for the gross commercial stand in the forest of the Jari.

\section{CONCLUSÕES}

Com base nos resultados obtidos, conclui-se que:

- Não foram encontradas diferenças significativas entre os tratamentos para as variáveis número de árvores total e número de árvores comerciais, considerando os 20 anos de monitoramento.

- As variáveis área basal e volume, de todas as espécies, apresentaram diferenças logo após a exploração e nos dois últimos períodos de avaliação, em conseqüência da aplicação dos tratamentos.

- Não foram encontradas diferenças estatísticas significativas, entre os tratamentos, para as variáveis área basal e volume de espécies comerciais, durante todo o período de acompanhamento.

- Considerando o período total de monitoramento, os tratamentos T1, T4, T5, T6, T9, T10, T11 e T12 tiveram balanço positivo, com o número de ingresso superior ao de mortalidade. Os tratamentos T2, T7 e T8 tiveram balanço negativo, e o tratamento T3 teve balanço nulo. Porém, as intervenções tiveram efeito significativo somente sobre a mortalidade. No povoamento comercial, mais de $50 \%$ dos tratamentos apresentaram balanço negativo ou nulo.

- Para o povoamento total líquido, o IPA $\mathrm{DAP}_{\mathrm{D}}$ apresentou as seguintes médias: tratamentos leves $=-0,04$ $\mathrm{cm}$ ano $^{-1} ;$ médio $=-0,12 \mathrm{~cm} \cdot$ ano $^{-1} ;$ e pesado $=-0,17 \mathrm{~cm} \cdot$ ano $^{-1}$. 
- O incremento periódico anual do diâmetro de espécies comerciais resultou em decrementos IPA $\mathrm{DAP}_{\mathrm{P}}$ $\left(-0,42 \mathrm{~cm} \cdot \mathrm{ano}^{-1}\right)$.

- No povoamento total bruto, o $\operatorname{IPA}_{\mathrm{DAP}}\left(0,30 \mathrm{~cm} \cdot \mathrm{ano}^{-1}\right)$ não foi favorecido pela extração e nem pelo desbaste.

- Considerando o povoamento comercial bruto, o $\operatorname{IPA}_{\mathrm{DAP}}\left(0,38 \mathrm{~cm} \cdot \mathrm{ano}^{-1}\right)$ variou significativamente somente em função do tempo.

- O baixo crescimento da floresta no tempo decorrido após a exploração e a aplicação dos desbastes indica que uma nova intervenção deve ser feita na área de estudo.

- Exploração de impacto reduzido mantém as funções ecológicas e ambientais da floresta, mas não, necessariamente, estimula o crescimento de espécies comerciais, e deve levar a ciclos de corte maiores do que os desejáveis trinta anos.

\section{AGRADECIMENTOS}

Ao Grupo Orsa, pelo apoio logístico na coleta dos dados. À Fundação de Amparo à Pesquisa do Estado do Amazonas (FAPEAM), pela bolsa de estudo.

\section{REFERÊNCIAS}

ALDER, D. Growth modelling for mixed tropical forests. Oxford, UK: University of Oxford. Department of Plant Sciences, 1995. 231 p. (Tropical Forestry Paper, n. 30).

COSTA, D. H. M.; SILVA, S. M. A. da; SILVA, J. N. M. Efetividade e custo do desbaste com aplicação de arboricida em floresta natural na região do Tapajós, Pará e Jarí, Amapá. In: SILVA, J. N. M.; CARVALHO, J. O. P. de; YARED, J. A. G. (Eds.) A silvicultura na Amazônia Oriental: contribuições do projeto Embrapa/DFID. Belém: Embrapa Amazônia Oriental/DFID, 2001. p.339-352.

CRUZ, C. D. Programa Genes: versão Windows; aplicativo computacional em genética e estatística. Viçosa, MG. UFV. 2001. 648 p.

DE GRAAF, N. R. A silvicultural system for natural regeneration of tropical rainforest in Suriname. Wageningem, The Netherlands: University of Wageningem. 1986. 250 p.

DE GRAAF, N. R.; POELS, R. L. H.; van ROMPAEY, R. S. A. R. Effect of silvicultural treatments on growth and mortality of rainforest in Suriname over long periods. Forest Ecology and Management. Amsterdam, v.124, n. 2-3, p.123-135, 1999.

DEKKER, M.; DE GRAAF, N. R. Pioneer and climax tree regeneration following selective logging with silviculture in Suriname. Forest Ecology and Management, Amsterdam, v.172, n.2-3, p.183- 190, 2003.

GOMIDE, G. L. A. Estrutura e dinâmica de crescimento de florestas tropicais primária e secundária no estado do Amapá. 179 f. Dissertação (Mestrado em Engenharia Florestal) - Setor de Ciências Agrárias, Universidade Federal do Paraná, Curitiba, 1997.

HIGUCHI, N.; SANTOS, J. dos; RIBEIRO, R. J.; FREITAS, J. V.; VIEIRA, G.; COIC, A. R.; MINETTE, L. J. Crescimento e incremento de uma floresta Amazônica de terra-firme manejada experimentalmente. In: Workshop Bionte - Aspectos Silviculturais e ecológicos do Manejo Florestal INPA - Manaus, 1997.

HIGUCHI, N.; VIEIRA, G.; MINETTE, L. J.; FREITAS, J. V.; JARDIM, F. C. S. Sistema SEL (Seleção de Espécies Listadas) para Manejar a Floresta Tropical Úmida de Terra-Firme da Amazônia In: VAL, A.L.; FRIGLIOULO, R.: FRIGLIOULO, FELDBERG, E. (Eds). Bases científicas para estratégias de preservação e desenvolvimento da Amazônia: fatos e perspectivas. Manaus: INPA, 1991. v. 1, p. 197-206.

JARDIM, F. C. S.; SOUZA, A. L.; BARROS, N. F.; MACHADO, C. C.; SILVA, E.; SILVA, A. F. Técnica de abertura do dossel por anelamento de árvores na Estação Experimental de Silvicultura do INPA, Manaus AM. Boletim daFaculdade de Ciências Agrárias do Pará, Belém, n. 25, p. 91-104, jan./jun., 1996. 
JONKERS, W. B. J. Vegetation structure logging damage and silviculture in a tropical rain forest in Suriname. Wageningen :Agricultural University, $1987.172 \mathrm{p}$.

LIEBERMAN, D.; LIEBERMAN, M. Forest tree growth and dynamics et La Selva, Costa Rica (19691982). Journal of tropical ecology, Cambridge, GB, n. 3, p. 347-358, 1987.

LOPES, J. C. A. Demografia e flutuações temporais da regeneração natural após uma exploração florestal: Flona Tapajós/PA. 133p. Dissertação (Mestrado) - Escola Superior de Agricultura "Luiz de Queiróz”, Universidade de São Paulo, Piracicaba, 1993.

MOSER, E. B.; SAXTON, A. M.; PEZESHKI. S. R. Repeated measures analysis of variance: application to tree research. Canadian Journal of Forest Research, Ottawa, v. 20, p. 524-535, 1990.

OLIVEIRA, L. C. Efeito da exploração da madeira e de diferentes intensidades de desbastes sobre a dinâmica da vegetação de uma área de 136 ha na Floresta Nacional do Tapajós. 195 p. Tese (Doutorado) - Escola Superior de Agricultura "Luiz de Queiroz", Universidade de São Paulo, Piracicaba, 2005.

PÉLLICO NETTO, S. BRENA, D. A. Inventário Florestal. Curitiba: Editado pelos autores. 1997. 316 p.

QUEIROZ, W. T. Técnicas de amostragem em inventário florestal nos Trópicos. Belém: FCAP. Serviço de Documentação e Informação. 1998. 147 p.

ROCHA, R. M. Taxas de recrutamento e mortalidade da floresta de terra firme da bacia do Rio Cuieiras na região de Manaus-AM. 49 p. Dissertação (Mestrado em Ciências Florestais) - Universidade Federal do Amazonas, Manaus, 2001.

SCOTT, A. J.; KNOTT, M. A. A cluster analysis method for grouping means in the analysis of variance. Biometrics, Washington, DC, v. 30, n. 2, p. 507-512, 1974.

SILVA, E. J. V. Dinâmica de florestas manejadas e sob exploração convencional na Amazônia oriental. 171 p. Tese (Doutorado em Ciências da Engenharia Ambiental) - Universidade de São Paulo, São Carlos, SP, 2004.

SILVA, J. N. M., ARAÚJO, S. M. Equação de volume para árvores de menor diâmetro na Floresta Nacional do Tapajós. Boletim de Pesquisa Florestal, Curitiba, v.8/9, p.16-25, 1984.

SILVA, J. N. M.; CARVAlHO, J. O. P. de; LOPES, J. do C. A.; CARVALHO, M. S. P. de. Equação de volume para a Floresta Nacional do Tapajós. Boletim de Pesquisa Florestal, Curitiba, v.8/9, p.50-63, 1984.

SILVA, J. N. M. The behaviour of the tropical rain forest of the Brazilian Amazon after logging. 303 p. PhD Thesis. University of Oxford, Oxford, GB, 1989.

SILVA, J. N. M.; de CARVAlHO, J. O. P.; LOPES, J. C. A.; de OLIVEIRA, R. P., de OLIVEIRA, L. C. Growth and yield studies in the Tapajós region, Central Brazilian Amazon. Commonwealth Forestry Review, Oxford, GB, v. 75, n. 4, p. 325-329, 1996.

Silva, J. N. M.; CARVAlhO, J. O. P.; LOPES, C. A.; AlMEIDA, B. F.; COSTA, D. H. M.; OLIVEIRA, L. C.; VANCLAY, J. K.; SKOVSGAARD, J. P. Growth and yield of a tropical rain forest in the Brazilian Amazon 13 year after logging. Forest Ecology and Management, Amsterdam, v. 71, n. 12, p. 267-274. 1995.

SILVA, J. N. M.; LOPES, J. C. A. Inventário florestal continuo em florestas tropicais: a metodologia utilizada pela EMBRAPA-CPATU na Amazônia brasileira. Belém: Embrapa-CPATU, 1984, 36 p. (Embrapa-CPATU. Documentos, n. 33).

SIlvA, S. M. A. da; SIlVA, J. N. M.; BAIMA, A. M. V.; LOBATO, N. M.; THOMPSON, I. S.; COSTA-FILHO, P. P. Impacto da exploração madeireira em floresta de terra firme no Município de Moju, Estado do Pará. In: SILVA, J. N. M.; CARVALHO, J. O. P. de; YARED, J. A. G. A silvicultura na Amazônia Oriental: contribuições do projeto Embrapa/DFID. Belém: Embrapa Amazônia Oriental/DFID, 2001. p. 309-323. 
SWAINE. M. D.; LIEBERMAN, D.; PUTZ, F. E. The dynamics of tree populations in tropical forest. Journal of Tropical Ecology, Cambridge, GB, v. 3, n. 4, p. 285-289, 1987.

VON ENDE, C. N. Repetead-Measures Analysis: Growth and Other Time-Dependent Measures. SCHEINER, S. M.; VINCENT, J. F. (Eds). Design and Analysis of Ecological Experiments. Yale University Press, 2001. p. $134-157$.

YARED, J. A. G.; CARVALHO, J. O P.; SILVA, J. N. M.; KANASHIRO, M.; MARQUES, L. C. T. Contribuições do Projeto Silvicultura Tropical: cooperação internacional Brasil/Reino Unido. Belém. Embrapa Amazônia Oriental/DEFID. 2000. 28 p. (Documentos, n. 52). 